TITLE:

\title{
Kinetic Theory of a Dilute Gas System under Steady Heat Conduction
}

\section{$\operatorname{AUTHOR}(\mathrm{S})$ :}

Kim, Hyeon-Deuk; Hayakawa, Hisao

\section{CITATION:}

Kim, Hyeon-Deuk ... [et al]. Kinetic Theory of a Dilute Gas System under Steady Heat Conduction. Journal of the Physical Society of Japan 2003, 72(8): 1904-1916

ISSUE DATE:

2003-08

URL:

http://hdl.handle.net/2433/49788

\section{RIGHT:}

Copyright (c) 2003 The Physical Society of Japan; This is not the

published version. Please cite only the published version.; この論文は

出版社版でありません。引用の際には出版社版をご確認ご利用くださ い。 


\title{
Addenda to "Kinetic Theory of a Dilute Gas System under Steady Heat Conduction"
}

\author{
Kim Hyeon-Deuk *† \\ Graduate School of Human and Environmental Studies, \\ Kyoto University, Kyoto 606-8501, Japan \\ Hisao Hayakawa $\ddagger$ \\ Department of Physics, Yoshida-South Campus, \\ Kyoto University, Kyoto 606-8501, Japan
}

PACS numbers: 05.20.Dd, 51.10.+y, 51.30.+i

\footnotetext{
*kim@yuragi.jinkan.kyoto-u.ac.jp

$\dagger$ Present address: Department of Chemistry, Kyoto University, Kyoto 606-8502

¥ hisao@yuragi.jinkan.kyoto-u.ac.jp
} 
We have derived the explicit velocity distribution function of the steady-state Boltzmann equation for hard-core molecules to second order in density and the temperature gradient. 1] We correct some mistakes in the results in this addenda.

The second-order coefficients $B_{k r}^{\mathrm{II}}$ in eq.(38) in our recent paper 1] should be written in the form:

$$
B_{k r}^{\mathrm{II}}=\frac{\delta_{k, 2}}{\pi \sigma^{4} n^{2} T^{2}}\left\{b_{2 r}^{A}\left[2\left(\partial_{z} T\right)^{2}-\left(\partial_{x} T\right)^{2}-\left(\partial_{y} T\right)^{2}\right]+b_{2 r}^{B} T\left[2 \partial_{z}^{2} T-\partial_{x}^{2} T-\partial_{y}^{2} T\right]\right\} .
$$

Values for the constants $b_{2 r}^{A}$ and $b_{2 r}^{B}$ are summarized in Table \ $\sigma, n, T$ are the diameter of hard-core molecules, density and temperature of gases, respectively. Owing to the properties of the spherical harmonic function[2], $B_{k r}^{(1) I I}$ can be obtained by replacing $2\left(\partial_{z} T\right)^{2}-\left(\partial_{x} T\right)^{2}-$ $\left(\partial_{y} T\right)^{2}$ and $2 \partial_{z}^{2} T-\partial_{x}^{2} T-\partial_{y}^{2} T$ in eq.(1) by $6 \partial_{z} T \partial_{x} T$ and $6 \partial_{z} \partial_{x} T$, respectively, using an axis change. Similarly, $C_{k r}^{(1) I I}$ can be obtained by replacing $2\left(\partial_{z} T\right)^{2}-\left(\partial_{x} T\right)^{2}-\left(\partial_{y} T\right)^{2}$ and $2 \partial_{z}^{2} T-\partial_{x}^{2} T-\partial_{y}^{2} T$ by $6 \partial_{z} T \partial_{y} T$ and $6 \partial_{z} \partial_{y} T$, respectively. $B_{k r}^{(2) \mathrm{II}}$ can be obtained by replacing $2\left(\partial_{z} T\right)^{2}-\left(\partial_{x} T\right)^{2}-\left(\partial_{y} T\right)^{2}$ and $2 \partial_{z}^{2} T-\partial_{x}^{2} T-\partial_{y}^{2} T$ by $6\left[\left(\partial_{x} T\right)^{2}-\left(\partial_{y} T\right)^{2}\right]$ and $6\left(\partial_{x}^{2} T-\right.$ $\left.\partial_{y}^{2} T\right)$, respectively, via an axis change; $C_{k r}^{(2) I I}$ by replacement by $12 \partial_{x} T \partial_{y} T$ and $12 \partial_{x} \partial_{y} T$, respectively.

The calculation of $B_{k r}^{\mathrm{II}}$ in Appendix D of our paper[1] should be also changed as follows. Equations (D.4), (D.5) and (D.6) become

$$
\begin{aligned}
\Omega_{20}^{\mathrm{H}} & =\frac{5}{16} \frac{1}{\sqrt{2 \pi} \sigma^{2} T^{2}}\left(\frac{m}{2 \kappa T}\right)^{-\frac{1}{2}}\left\{-\frac{b_{11}}{2}\left[2\left(\partial_{z} T\right)^{2}-\left(\partial_{x} T\right)^{2}-\left(\partial_{y} T\right)^{2}\right]\right. \\
& \left.-b_{11} T\left[2 \partial_{z}^{2} T-\partial_{x}^{2} T-\partial_{y}^{2} T\right]\right\}
\end{aligned}
$$

and

$$
\begin{aligned}
\Omega_{21}^{\mathrm{H}} & =\frac{5}{16} \frac{1}{\sqrt{2 \pi} \sigma^{2} T^{2}}\left(\frac{m}{2 \kappa T}\right)^{-\frac{1}{2}} \\
& \times\left\{\left[2\left(\partial_{z} T\right)^{2}-\left(\partial_{x} T\right)^{2}-\left(\partial_{y} T\right)^{2}\right]\left[\frac{5}{2} b_{11}-3 b_{12}\right]\right. \\
& \left.+T\left[2 \partial_{z}^{2} T-\partial_{x}^{2} T-\partial_{y}^{2} T\right]\left[b_{11}-2 b_{12}\right]\right\},
\end{aligned}
$$

and

$$
\begin{aligned}
\Omega_{2 r}^{\mathrm{H}} & =\frac{5}{16} \frac{1}{\sqrt{2 \pi} \sigma^{2} T^{2}}\left(\frac{m}{2 \kappa T}\right)^{-\frac{1}{2}} \\
& \times\left\{\left[2\left(\partial_{z} T\right)^{2}-\left(\partial_{x} T\right)^{2}-\left(\partial_{y} T\right)^{2}\right]\left[-(r+1)\left(r+\frac{1}{2}\right) b_{1, r+1}+\left(2 r+\frac{1}{2}\right) b_{1 r}-b_{1, r-1}\right]\right. \\
& \left.+T\left[2 \partial_{z}^{2} T-\partial_{x}^{2} T-\partial_{y}^{2} T\right]\left[-(r+1) b_{1, r+1}+b_{1 r}\right]\right\},
\end{aligned}
$$


respectively. Here $m$ and $\kappa$ are the mass of hard-core molecules and the Boltzmann constant. No other corrections appear in the calculation of $B_{k r}^{\mathrm{II}}$ in Appendix D of our paper[1]. We emphasize that the final form and the numerical values $b_{1 r}, b_{0 r}$ and $b_{2 r}$ of the velocity distribution function to second order in eq.(39) of our paper 1] which we have used to calculate thermodynamic quantities are unchanged. We also note that the 4 th approximation $b_{20}$ in Table III of our paper 1] still deviates from Burnett's 4th approximation $b_{20}$ by a factor 1.003. 2] This deviation is considered to be due to errors in Burnett's calculation, as was indicated in our paper [1]. Furthermore, we note a miss print in eq. (A.4) of our recent paper[1]. Its correct form is

$$
G_{k, r}=\frac{k+1}{2 k+3}\left\{\left(k+r+\frac{3}{2}\right) B_{k+1, r}-B_{k+1, r-1}\right\}+\frac{k}{2 k-1}\left\{B_{k-1, r}-(r+1) B_{k-1, r+1}\right\} .
$$

Finally, we correct the numerical values $\lambda_{T_{\mathrm{i}}}$ in each component of the kinetic temperature $T_{\mathrm{i}}$ for $\mathrm{i}=x, y$ and $z$. In eq.(44) of our paper [1], the values of $\lambda_{T_{\mathrm{i}}}$ should be identical with those of $\lambda_{P}^{i i}$ in Table IV of the paper [1]. We also indicate that the second-order pressure tensor in eq.(43) of our paper[1] should be uniform from the solubility conditions for the third-order solution $\phi_{1}^{(3)}$.

[1] Kim H.-D. and H. Hayakawa: J. Phys. Soc. Jpn. 72 (2003) 1904.

[2] D. Burnett: Proc. Lond. Math. Soc. 40 (1935) 382. 
TABLE I: Numerical constants $b_{2 r}^{A}$ (upper) and $b_{2 r}^{B}$ (lower) in eq.(11)

\begin{tabular}{lcccc}
\hline$r$ & $r \leq 4$ & $r \leq 5$ & $r \leq 6$ & $r \leq 7$ \\
\hline 0 & $7.065 \times 10^{-3}$ & $7.312 \times 10^{-3}$ & $7.366 \times 10^{-3}$ & $7.380 \times 10^{-3}$ \\
\hline $1-1.654 \times 10^{-1}$ & $-1.647 \times 10^{-1}$ & $-1.646 \times 10^{-1}$ & $-1.646 \times 10^{-1}$ \\
\hline 2 & $5.970 \times 10^{-2}$ & $6.040 \times 10^{-2}$ & $6.055 \times 10^{-2}$ & $6.059 \times 10^{-2}$ \\
\hline 3 & $4.593 \times 10^{-3}$ & $5.071 \times 10^{-3}$ & $5.166 \times 10^{-3}$ & $5.190 \times 10^{-3}$ \\
\hline 4 & - & $3.364 \times 10^{-4}$ & $3.824 \times 10^{-4}$ & $3.930 \times 10^{-4}$ \\
\hline 5 & - & - & $2.495 \times 10^{-5}$ & $2.888 \times 10^{-5}$ \\
\hline 6 & - & - & - & $1.726 \times 10^{-6}$ \\
\hline & & & & \\
\hline$r$ & $r \leq 4$ & & & \\
\hline 0 & $8.119 \times 10^{-2}$ & $8.117 \times 10^{-2}$ & $8.116 \times 10^{-2}$ & $8.116 \times 10^{-2}$ \\
\hline $1-7.380 \times 10^{-2}$ & $-7.388 \times 10^{-2}$ & $-7.390 \times 10^{-2}$ & $-7.390 \times 10^{-2}$ \\
\hline $2-7.001 \times 10^{-3}$ & $-7.090 \times 10^{-3}$ & $-7.108 \times 10^{-3}$ & $-7.113 \times 10^{-3}$ \\
\hline 3 & $5.828 \times 10^{-4}$ & $6.469 \times 10^{-4}$ & $-6.595 \times 10^{-4}$ & $-6.626 \times 10^{-4}$ \\
\hline 4 & - & $-4.897 \times 10^{-5}$ & $-5.541 \times 10^{-5}$ & $-5.687 \times 10^{-5}$ \\
\hline 5 & - & - & $-3.796 \times 10^{-6}$ & $-4.365 \times 10^{-6}$ \\
\hline 6 & - & - & $-2.716 \times 10^{-6}$ \\
\hline
\end{tabular}




\title{
Kinetic Theory of a Dilute Gas System under Steady Heat Conduction
}

\author{
Kim Hyeon-Deuk * \\ Graduate School of Human and Environmental Studies, \\ Kyoto University, Kyoto 606-8501, Japan \\ Hisao Hayakawa ${ }^{\dagger}$ \\ Department of Physics, Yoshida-South Campus, \\ Kyoto University, Kyoto 606-8501, Japan
}

(Dated: February 26, 2008)

\begin{abstract}
The velocity distribution function of the steady-state Boltzmann equation for hard-core molecules in the presence of a temperature gradient has been obtained explicitly to second order in density and the temperature gradient. Some thermodynamical quantities are calculated from the velocity distribution function for hard-core molecules and compared with those for Maxwell molecules and the steady-state Bhatnagar-Gross-Krook(BGK) equation. We have found qualitative differences between hard-core molecules and Maxwell molecules in the thermodynamical quantities, and also confirmed that the steady-state BGK equation belongs to the same universality class as Maxwell molecules.
\end{abstract}

PACS numbers: 05.20.Dd, 51.10.+y, 51.30.+i

\footnotetext{
* kim@yuragi.jinkan.kyoto-u.ac.jp

† hisao@yuragi.jinkan.kyoto-u.ac.jp
} 


\section{INTRODUCTION}

The kinetic theory has long history and various investigations have been carried out analytically, numerically and experimentally. [1, 2, 3, 4, 4, 5, 6] Text books on the Boltzmann equation [7, 8, 9, 10, 11] show the fact that the Boltzmann equation has attracted much interest among researchers of kinetic phenomena. It is well accepted that the Boltzmann equation is one of the most reliable kinetic models for describing nonequilibrium phenomena.

A number of studies on the Boltzmann equation have been based on two representative models of molecules: hard-core molecules and Maxwell molecules [12, 13, 14]. Maxwell molecules interact via a force that is inversely proportional to the fifth power of the distance $r$, that is, Maxwell molecules interact via a central potential proportional to $r^{-4}$. It is well known that calculations involving the Boltzmann equation for Maxwell molecules become much easier than those for hard-core molecules. 11, 12, 13, 14, 15, 16, 17] In order to obtain the velocity distribution function of a dilute gas system in a nonequilibrium state, various methods, such as the Chapman-Enskog method, the Grad method and the Hilbert method, have been presented. 11, 2, 3, 4, 5, 6, 7, 8, 9, 10, 11, 14, 15, 16, 17, 18, 19, 20] They are well known as methods to derive the normal solutions of the Boltzmann equation.

Other kinetic models besides the Boltzmann equation, such as the linearized Boltzmann equation 5, 21] and the Bhatnagar-Gross-Krook (BGK) equation [22, 23, 24, 25, 26, 27, 28, 29, 30, 31, 32, 33], have also been proposed in order to avoid mathematical difficulties in dealing with the collision term of the Boltzmann equation. It has been believed that results of those kinetic models approximately agree with those of the Boltzmann equation, though they are accepted as quantitatively different ones from the Boltzmann equation: e.g. the Prandtl number is $2 / 3$ for the Boltzmann equation, while it is 1 for the BGK equation. 24, 25] The BGK equation retains important properties of the Boltzmann equation, such as the conservation laws and the H-theorem. It has been felt that characteristics of molecules, such as hard-core molecules and Maxwell molecules, can be absorbed into the relaxation time $\tau$. The most important contribution concerning the solution of the BGK equation has been made by Santos and his coworkers. 26, 27, 28, 29, 30, 31, 32, 33] They have solved the steady-state BGK equation exactly and compared its exact solution with the Chapman-Enskog solution derived to arbitrary order. [26, 27, 28, 29, 30] They have concluded that the Chapman-Enskog solution is asymptotically correct, although the 
Chapman-Enskog series diverge. [26, 27, 28, 29, 30] They have also compared high order velocity distribution function for the steady-state BGK equation with that for the Boltzmann equation for Maxwell molecules under uniform shear flow. [31, 32, 33]

On the other hand, it had been believed that Burnett [15] determined the complete second order solution of the Boltzmann equation by the Chapman-Enskog method. 1] Importance of the second-order coefficients has been demonstrated for descriptions of shock wave profiles and sound propagation phenomena. [34, 35, 36, 37] However, we have realized that Burnett's solution [15] is not complete. Though there are various studies on the transport coefficients of the Boltzmann equation to second order [1, 2, 3, 19, 20, 38, 39] or even to third order [34], we have found that nobody has derived the explicit velocity distribution function of the Boltzmann equation for hard-core molecules to second order. This is a result of mathematical difficulties, as was indicated by Fort and Cukrowski[40]. For Maxwell molecules, Schamberg [16] has derived the precise velocity distribution function of the Boltzmann equation to second order by the Chapman-Enskog method, while Shavaliev[17] has derived it implicitly by the moment method.

In this paper, we derive the explicit velocity distribution function of the steady-state Boltzmann equation for hard-core molecules to second order in density and temperature gradients by the Chapman-Enskog method. The derivation of it is of some physical significance. For example, it has been required for calculating a nonequilibrium effect on the rate of chemical reaction [4], because the nonequilibrium effect on the rate of chemical reaction does not appear to first order and it cannot be calculated by the moment method. [41] There also exists a need to confirm the existence of universal nonlinear nonequilibrium statistical mechanics from the microscopic viewpoint as mentioned in ref. [40], so that it is necessary to derive the explicit velocity distribution function of the steady-state Boltzmann equation for hard-core molecules to second order and compare it with that for Maxwell molecules. Additionally, the derivation of the explicit second-order solution of the steady-state Boltzmann equation for hard-core molecules by the Chapman-Enskog method will contribute to an understanding of the difference or the correspondence between the Chapman-Enskog method and the Grad method by the direct comparison of the solution by the Chapman-Enskog method with that by the Grad method. In the present paper, we also discuss the relation between the steady-state Boltzmann equation and the steady-state BGK equation which has not been fully understood yet. 
The organization of this paper is as follows. In \$II we will introduce the ChapmanEnskog method to solve the steady state Boltzmann equation. In explicit form of the velocity distribution function of the steady-state Boltzmann equation for hard-core molecules to second order in the density and temperature gradients. In particular, the result for the first-order coefficients is shown in eq.(33), and the results for the secondorder coefficients are shown in eqs.(37) and (38). The velocity distribution function of the steady-state Boltzmann equation to second order for hard-core molecules is shown explicitly in eq.(39) and graphically in Fig,2, and compared directly with that for Maxwell molecules (40) in Fig [3, In §IV] we will apply the velocity distribution function to second order for hardcore molecules (39) and those for Maxwell molecules (40) and the steady-state BGK equation to a nonequilibrium steady-state system under steady heat conduction and calculate some thermodynamical quantities. We stress the existence of the qualitative differences among hard-core molecules, Maxwell molecules and the steady-state BGK equation in the pressure tensor (43) and the kinetic temperature (44). Our discussion and conclusion are written in $\S$ V and VI respectively.

\section{METHOD FOR SOLVING THE STEADY-STATE BOLTZMANN EQUATION}

Let us introduce the Chapman-Enskog method to solve the steady-state Boltzmann equation in this section. Assume that we have a system of dilute gases in a steady state, with velocity distribution function $f_{1}=f\left(\mathbf{r}, \mathbf{v}_{1}\right)$. The appropriate steady-state Boltzmann equation is

$$
\mathbf{v}_{1} \cdot \nabla f_{1}=J\left(f_{1}, f_{2}\right)
$$

where the collision integral $J\left(f_{1}, f_{2}\right)$ is expressed as

$$
J\left(f_{1}, f_{2}\right)=\iiint\left(f_{1}^{\prime} f_{2}^{\prime}-f_{1} f_{2}\right) g b \mathrm{~d} b \mathrm{~d} \epsilon \mathrm{d} \mathbf{v}_{2}
$$

with $f_{1}^{\prime}=f\left(\mathbf{r}, \mathbf{v}_{1}^{\prime}\right)$ and $f_{2}^{\prime}=f\left(\mathbf{r}, \mathbf{v}_{2}^{\prime}\right): \mathbf{v}_{1}^{\prime}$ and $\mathbf{v}_{2}^{\prime}$ are postcollisional velocities of $\mathbf{v}_{1}$ and $\mathbf{v}_{2}$, respectively. The relative velocity of two molecules before and after the interaction has the same magnitude $g=\left|\mathbf{v}_{1}-\mathbf{v}_{2}\right|$; the angle between the directions of the relative velocity before and after the interaction is represented by $\chi$. The relative position of the two molecules is represented by $b$, called the impact parameter, and $\epsilon$ represents the orientation of the plane in which $\mathbf{g}$ and $\mathbf{g}^{\prime}=\mathbf{v}_{1}^{\prime}-\mathbf{v}_{2}^{\prime}$ belong. The impact parameter $b$ depends on the kind of 
interactions between molecules. Note that $\chi$ can be expressed as a function of $b$ for a central force. (see Fig (1)

Suppose that the velocity distribution function $f_{1}$ can be expanded as:

$$
f_{1}=f_{1}^{(0)}+f_{1}^{(1)}+f_{1}^{(2)}+\cdots=f_{1}^{(0)}\left(1+\phi_{1}^{(1)}+\phi_{1}^{(2)}+\cdots\right) .
$$

$f_{1}^{(0)}$ is the local Maxwellian distribution function, written as

$$
f_{1}^{(0)}=n(\mathbf{r})\left(\frac{m}{2 \pi \kappa T(\mathbf{r})}\right)^{\frac{3}{2}} \exp \left[-\frac{m \mathbf{v}_{1}^{2}}{2 \kappa T(\mathbf{r})}\right],
$$

with $m$ mass of the molecules and $\kappa$ the Boltzmann constant. $n(\mathbf{r})$ and $T(\mathbf{r})$ will be identified later as the density and the temperature at position $\mathbf{r}$, respectively. Substituting eq.(3) into the steady-state Boltzmann equation (11), we arrive at the following set of equations which we will solve completely in this paper:

$$
L\left[f_{1}^{(0)}\right] \phi_{1}^{(1)}=\mathbf{v}_{1} \cdot \nabla f_{1}^{(0)}
$$

to first order and

$$
L\left[f_{1}^{(0)}\right] \phi_{1}^{(2)}=\mathbf{v}_{1} \cdot \nabla f_{1}^{(1)}-J\left(f_{1}^{(1)}, f_{2}^{(1)}\right),
$$

to second order. The linear integral operator $L\left[f_{1}^{(0)}\right]$ is defined as

$$
L\left[f_{1}^{(0)}\right] X_{1} \equiv \iiint f_{1}^{(0)} f_{2}^{(0)}\left(X_{1}^{\prime}-X_{1}+X_{2}^{\prime}-X_{2}\right) g b \mathrm{~d} b \mathrm{~d} \epsilon \mathrm{d} \mathbf{v}_{2} .
$$

The solubility conditions of the integral equation (15) are given by

$$
\int \Phi_{\mathrm{i}} \mathbf{v}_{1} \cdot \nabla f_{1}^{(0)} \mathrm{d} \mathbf{v}_{1}=0
$$

where $\Phi_{\mathrm{i}}$ is one of the collisional invariants:

$$
\Phi_{1}=1, \quad \Phi_{2}=m \mathbf{v}_{1}, \quad \Phi_{3}=\frac{1}{2} m \mathbf{v}_{1}^{2}
$$

Substituting eq.(41) into the solubility conditions (8), it is seen that $n \kappa T$ is uniform in the steady state. We use this result in our calculation. Similarly, the solubility conditions of the integral equation ([6) are given by

$$
\int \Phi_{\mathrm{i}} \mathbf{v}_{1} \cdot \nabla f_{1}^{(1)} \mathrm{d} \mathbf{v}_{1}=0
$$


To construct solutions of the integral equations (5) and (6) definite, five further conditions must be specified; we identify the density:

$$
n(\mathbf{r}) \equiv \int f_{1} \mathrm{~d} \mathbf{v}_{1}=\int f_{1}^{(0)} \mathrm{d} \mathbf{v}_{1}
$$

the temperature:

$$
\frac{3 n(\mathbf{r}) \kappa T(\mathbf{r})}{2} \equiv \int \frac{m \mathbf{v}_{1}^{2}}{2} f_{1} \mathrm{~d} \mathbf{v}_{1}=\int \frac{m \mathbf{v}_{1}^{2}}{2} f_{1}^{(0)} \mathrm{d} \mathbf{v}_{1}
$$

and the mean flow:

$$
\mathbf{C}_{0} \equiv \int m \mathbf{v}_{1} f_{1} \mathrm{~d} \mathbf{v}_{1}=\int m \mathbf{v}_{1} f_{1}^{(0)} \mathrm{d} \mathbf{v}_{1}
$$

Here we assume that no mean flow, i.e. $\mathbf{C}_{0}=0$, exists in the system. The introduction of these conditions distinguishes the Chapman-Enskog adopted here from the Hilbert method in which the conserved quantities are also expanded. 2] We assert that conditions (11), (12) and (13) do not affect all our results in this paper. It should be noted that, to solve the integral equations (5) and (6), we should consider only the case in which the right-hand sides of eqs.(15) and (6) are not zero: if the right-hand sides of eqs.(15) and (6) are zero, the integral equations (51) and (6) become homogeneous equations which do not have any particular solutions. [2]

\section{BURNETT'S METHOD}

\section{A. A general form of the velocity distribution function}

To solve the integral equations (5) and (6), Burnett 15] assumed a general form of the velocity distribution function:

$$
f_{1}=f_{1}^{(0)}\left[\sum_{r=0}^{\infty} r ! \Gamma\left(r+\frac{3}{2}\right) B_{0 r} S_{\frac{1}{2}}^{r}\left(\mathbf{c}_{1}^{2}\right)+\sum_{k=1}^{\infty}\left(\frac{m}{2 \kappa T}\right)^{\frac{k}{2}} \sum_{r=0}^{\infty} r ! \Gamma\left(k+r+\frac{3}{2}\right) Y_{k r}\left(\mathbf{c}_{1}\right) S_{k+\frac{1}{2}}^{r}\left(\mathbf{c}_{1}^{2}\right)\right] .
$$

Here $\mathbf{c}_{1} \equiv(m / 2 \kappa T)^{1 / 2} \mathbf{v}_{1}$ is the scaled velocity and $\Gamma(X)$ represents the Gamma function. $S_{k}^{p}(X)$ is a Sonine polynomial, defined by

$$
(1-\omega)^{-k-1} e^{-\frac{X \omega}{1-\omega}}=\sum_{p=0}^{\infty} \Gamma(p+k+1) S_{k}^{p}(X) \omega^{p}
$$

$Y_{k r}\left(\mathbf{c}_{1}\right)$ is a linear combination of spherical harmonic functions:

$$
Y_{k r}\left(\mathbf{c}_{1}\right)=B_{k r} Y_{k}\left(\mathbf{c}_{1}\right)+2 \sum_{p=1}^{k} \frac{(k-p) !}{(k+p) !}\left[B_{k r}^{(p)} Y_{k}^{(p)}\left(\mathbf{c}_{1}\right)+C_{k r}^{(p)} Z_{k}^{(p)}\left(\mathbf{c}_{1}\right)\right]
$$


where $B_{k r}, B_{k r}^{(p)}$ and $C_{k r}^{(p)}$ are coefficients to be determined. Introducing the normal spherical coordinate representation for $\mathbf{c}_{1}$, i.e. $c_{1 x}=c_{1} \sin \theta \cos \phi, c_{1 y}=c_{1} \sin \theta \sin \phi$ and $c_{1 z}=c_{1} \cos \theta$, the spherical harmonic functions $Y_{k}\left(\mathbf{c}_{1}\right), Y_{k}^{(p)}\left(\mathbf{c}_{1}\right)$ and $Z_{k}^{(p)}\left(\mathbf{c}_{1}\right)$ are expressed as

$$
Y_{k}\left(\mathbf{c}_{1}\right)=\left(\frac{2 \kappa T}{m}\right)^{\frac{k}{2}} c_{1}^{k} P_{k}(\cos \theta),
$$

and

$$
Y_{k}^{(p)}\left(\mathbf{c}_{1}\right)=(-1)^{p}\left(\frac{2 \kappa T}{m}\right)^{\frac{k}{2}} c_{1}^{k} P_{k}^{(p)}(\cos \theta) \cos p \phi
$$

and

$$
Z_{k}^{(p)}\left(\mathbf{c}_{1}\right)=(-1)^{p}\left(\frac{2 \kappa T}{m}\right)^{\frac{k}{2}} c_{1}^{k} P_{k}^{(p)}(\cos \theta) \sin p \phi
$$

with the Legendre polynomial $P_{k}(\cos \theta)$ and the associated Legendre polynomial $P_{k}^{(p)}(\cos \theta)$.

Assumption of the velocity distribution function of eq.(14) has some mathematical advantages in our calculation. Firstly, it is sufficient to determine the coefficients $B_{k r}$, because $B_{k r}^{(p)}$ and $C_{k r}^{(p)}$ can be always determined from $B_{k r}$ by a transformation of axes owing to the properties of the spherical harmonic functions (17), (18) and (19). We call $B_{k r}, B_{k r}^{(p)}$ and $C_{k r}^{(p)}$ the family of $B_{k r}$. Secondly, some important physical quantities are related to coefficients $B_{k r}, B_{k r}^{(p)}, C_{k r}^{(p)}:$ e.g. the density (11), the temperature (12) and the mean flow (13) with $f_{1}$ in eq.(14) lead to the five equivalent conditions [15, 16]:

$$
B_{00}=1, \quad B_{10}=B_{10}^{(1)}=C_{10}^{(1)}=0, \quad B_{01}=0 .
$$

Similarly, the pressure tensor $P_{i j}$ defined by

$$
P_{i j}=\left(\frac{2 \kappa T}{m}\right)^{\frac{5}{2}} \int_{-\infty}^{\infty} \mathrm{d} \mathbf{c}_{1} m c_{1 i} c_{1 j} f_{1}
$$

for $i, j=x, y$ and $z$ is related to the family of $B_{20}$, which is the only family in which Burnett was interested. 15$]$

The coefficients $B_{k r}$ except for those in eq.(20) can be calculated as follows. Multiplying the steady-state Boltzmann equation (11) by

$$
Q_{k r}\left(\mathbf{c}_{1}\right) \equiv\left(k+\frac{1}{2}\right) \sqrt{\pi}\left(\frac{m}{2 \kappa T}\right)^{\frac{k}{2}} Y_{k}\left(\mathbf{c}_{1}\right) S_{k+\frac{1}{2}}^{r}\left(\mathbf{c}_{1}^{2}\right),
$$


and then integrating over $(2 \kappa T / m)^{1 / 2} \mathbf{c}_{1}$, it is found that

$$
\begin{array}{r}
-\left(\frac{2 \kappa T}{m}\right)^{\frac{1}{2}}<\mathbf{c}_{1} \cdot \nabla Q_{k r}>_{a v}+\nabla \cdot\left[\left(\frac{2 \kappa T}{m}\right)^{\frac{1}{2}}<\mathbf{c}_{1} Q_{k r}>_{a v}\right]= \\
\left(\frac{2 \kappa T}{m}\right)^{3} \iiint \int\left(Q_{k r}^{\prime}-Q_{k r}\right) f_{1} f_{2} g b \mathrm{~d} b \mathrm{~d} \epsilon \mathrm{d} \mathbf{c}_{2} \mathrm{~d} \mathbf{c}_{1},
\end{array}
$$

where $\left\langle X>_{a v}\right.$ represents $(2 \kappa T / m)^{3 / 2} \int X f_{1} \mathrm{~d} \mathbf{c}_{1}$, and $Q_{k r}^{\prime}$ represents the postcollisional $Q_{k r}$. We should calculate both sides of eq.(23) for every $k$ and $r$, because eq.(23) leads to equations to determine $B_{k r}$, as will be shown in Appendices $\mathrm{C}$ and $\mathrm{D}$.

For convenience, we introduce $\Omega_{k r}$ and $\Delta_{k r}$ as the left-hand and right-hand sides of eq.(23), respectively, i.e.

$$
\Omega_{k r} \equiv-\left(\frac{2 \kappa T}{m}\right)^{\frac{1}{2}}<\mathbf{c}_{1} \cdot \nabla Q_{k r}>_{a v}+\nabla \cdot\left[\left(\frac{2 \kappa T}{m}\right)^{\frac{1}{2}}<\mathbf{c}_{1} Q_{k r}>_{a v}\right]
$$

and

$$
\Delta_{k r} \equiv\left(\frac{2 \kappa T}{m}\right)^{3} \iiint \int\left(Q_{k r}^{\prime}-Q_{k r}\right) f_{1} f_{2} g b \mathrm{~d} b \mathrm{~d} \epsilon \mathrm{d} \mathbf{c}_{2} \mathrm{~d} \mathbf{c}_{1}
$$

We will calculate $\Omega_{k r}$ and $\Delta_{k r}$ separately. The result of $\Omega_{k r}$ becomes

$$
\begin{aligned}
\Omega_{k r} & =\frac{n}{T}\left(\frac{2 \kappa T}{m}\right)^{\frac{1}{2}}\left[\left(r+\frac{k}{2}\right)\left(D_{k, r} \partial_{x} T+E_{k, r} \partial_{y} T+G_{k, r} \partial_{z} T\right)\right. \\
& \left.-\left(D_{k, r-1} \partial_{x} T+E_{k, r-1} \partial_{y} T+G_{k, r-1} \partial_{z} T\right)\right] \\
& +\partial_{x}\left[n\left(\frac{2 \kappa T}{m}\right)^{\frac{1}{2}} D_{k, r}\right]+\partial_{y}\left[n\left(\frac{2 \kappa T}{m}\right)^{\frac{1}{2}} E_{k, r}\right]+\partial_{z}\left[n\left(\frac{2 \kappa T}{m}\right)^{\frac{1}{2}} G_{k, r}\right],
\end{aligned}
$$

where $\partial_{\mathrm{i}} X$ denotes $\partial X / \partial \mathrm{i}$ for $\mathrm{i}=x, y$ and $z . D_{k, r}, E_{k, r}$ and $G_{k, r}$ are functions of the family of $B_{k r}$, as is written in Appendix $\mathrm{A}$.

\section{B. The collision term $\Delta_{k r}$}

Next, we calculate the collision term $\Delta_{k r}$ in eq.(25). We should specify the kind of interactions of molecules so as to perform the calculation of the collision term $\Delta_{k r}$; the impact parameter $b$ is explicitly determined by specifying the type of interaction. For hardcore molecules, the impact parameter $b$ is given by the relation

$$
b=\sigma \cos \frac{\chi}{2}
$$


where $\sigma$ is the hard-core molecular diameter and $\chi$ is the scattering angle (see Fig 1). Therefore, $\Delta_{k r}$ for hard-core molecules, i.e. $\Delta_{k r}^{\mathrm{H}}$, becomes 15$]$

$$
\Delta_{k r}^{\mathrm{H}}=\frac{\sigma^{2}}{2} \int_{0}^{\pi}\left[F_{k r}^{1}(\chi)-F_{k r}^{1}(0)\right] \sin \frac{\chi}{2} \cos \frac{\chi}{2} \mathrm{~d} \chi,
$$

where $F_{k r}^{\mu}(\chi)$ is defined as

$$
F_{k r}^{\mu}(\chi) \equiv\left(\frac{2 \kappa T}{m}\right)^{3} \iiint Q_{k r}^{\prime} f_{1} f_{2} g^{\mu} \mathrm{d} \epsilon \mathrm{d} \mathbf{c}_{2} \mathrm{~d} \mathbf{c}_{1},
$$

and we have used $F_{k r}^{\mu}(0)=(2 \kappa T / m)^{3} \iiint Q_{k r} f_{1} f_{2} g^{\mu} \mathrm{d} \epsilon \mathrm{d} \mathbf{c}_{2} \mathrm{~d} \mathbf{c}_{1}$. Note that $\chi=0$ if $b>\sigma$. For Maxwell molecules, the impact parameter $b$ is given by the relation

$$
b \mathrm{~d} b=\frac{1}{g} H(\chi) \mathrm{d} \chi,
$$

where $H(\chi)$ is a function of $\chi \cdot[6,12,13,14]$ Therefore, $\Delta_{k r}$ for Maxwell molecules, i.e. $\Delta_{k r}^{\mathrm{M}}$, becomes

$$
\Delta_{k r}^{\mathrm{M}}=\int_{0}^{\pi}\left[F_{k r}^{0}(\chi)-F_{k r}^{0}(0)\right] H(\chi) \mathrm{d} \chi
$$

Since $\Delta_{k r}^{\mathrm{M}}$ has been calculated by Schamberg[16], we will calculate only $\Delta_{k r}^{\mathrm{H}}$ in this paper. Schamberg's result for $\Delta_{k r}^{\mathrm{M}}$ is briefly summarized in our web page. [42]

From eq.(28), it is sufficient to calculate $F_{k r}^{1}(\chi)$ for $\Delta_{k r}^{\mathrm{H}}$. The details of $F_{k r}^{1}(\chi)$ are written in Appendix B. Several explicit forms of $\Delta_{k r}^{\mathrm{H}}$ are also demonstrated in Appendices $\mathrm{C}$ and D. From the definitions (24) and (25), both sides of eq.(23) for arbitrary $k$ and $r$ can be calculated for hard-core molecules via

$$
\Omega_{k r}^{\mathrm{H}}=\Delta_{k r}^{\mathrm{H}}
$$

which produces a set of simultaneous equations determining the coefficients $B_{k r}$, as is explained in Appendices $\mathrm{C}$ and D. Here $\Omega_{k r}^{\mathrm{H}}$ denotes $\Omega_{k r}$ in eq.(26) for hard-core molecules.

\section{Determination of $B_{k r}$}

We will determine the first-order coefficients $B_{k r}^{\mathrm{I}}$ and the second-order coefficients $B_{k r}^{\mathrm{II}}$ in accordance with the previous two subsections, which corresponds to solving the integral equations (5) and (6) , respectively. Here the upper suffices I and II are introduced to specify the order of $B_{k r}$. We have provided an example of our Mathematica program for calculating these coefficients. 42$]$ 


\section{The first order}

We show the results of the first-order coefficients $B_{k r}^{\mathrm{I}}$ of which the solution of the integral equation (5), $\phi_{1}^{(1)}$, is composed. They can be written in the form:

$$
B_{k r}^{\mathrm{I}}=\delta_{k, 1} b_{1 r} \frac{15}{16} \frac{\partial_{z} T}{\sqrt{2 \pi} \sigma^{2} n T}
$$

Values of the constants $b_{1 r}$ are given in Table I. The calculation of $B_{k r}^{\mathrm{I}}$ is explained in Appendix C] It is seen that $B_{k r}^{\mathrm{I}}$ is of the order of the Knudsen number $K$, which means that the mean free path of molecules should be much less than the characteristic length for changes in the macroscopic variables. Though Burnett derived $B_{k r}^{\mathrm{I}}$ only to 4 th approximation, i.e. $B_{k r}^{\mathrm{I}}$ for $r \leq 4$, we have obtained $B_{k r}^{\mathrm{I}}$ for $r \leq 7$ in this paper. This ensures the convergence of all the physical quantities calculated in this paper. It should be mentioned that our values of $B_{k r}^{\mathrm{I}}$ for $r \leq 4$ agree with Burnett's values 15$]$. Once $B_{k r}^{\mathrm{I}}$ have been calculated, $B_{k r}^{(1) \mathrm{I}}$ can be written down directly by replacing $\partial_{z} T$ by $\partial_{x} T$ by symmetry, owing to the properties of the spherical harmonic function[15]; $C_{k r}^{(1) \mathrm{I}}$ can also be obtained similarly by replacing $\partial_{z} T$ by $\partial_{y} T$. Note that other terms, such as $B_{k r}^{(2) \mathrm{I}}$, do not appear because $p$ in $B_{k r}^{(p) \mathrm{I}}$ must be $k$ or less from eq.(16). Substituting all the first-order coefficients derived here into eq.(16), we can finally obtain the first-order velocity distribution function $f_{1}^{(1)}$.

\section{Solubility conditions for $\phi_{1}^{(2)}$}

Since the first-order velocity distribution function $f_{1}^{(1)}$ has been obtained, the solubility conditions of the integral equation (6) should be considered before we attempt to derive an expression for $\phi_{1}^{(2)}$. The solubility conditions for $\phi_{1}^{(2)}$, that is, eqs.(10) lead to the condition

$$
\nabla \cdot \mathbf{J}^{(1)}=0
$$

where $\mathbf{J}^{(1)}$, i.e. the heat flux for $f_{1}^{(1)}$, can be obtained as

$$
\begin{aligned}
\mathbf{J}^{(1)} & \equiv\left(\frac{2 \kappa T}{m}\right)^{3} \int_{-\infty}^{\infty} \mathrm{d} \mathbf{c}_{1} \frac{m \mathbf{c}_{1}^{2}}{2} \mathbf{c}_{1} f_{1}^{(1)} \\
& =-b_{11} \frac{75}{64}\left(\frac{\kappa T}{\pi m}\right)^{\frac{1}{2}} \frac{\kappa}{\sigma^{2}} \nabla T
\end{aligned}
$$

with the appropriate value for $b_{11}$ listed in Table II It must be emphasized that, since $\mathbf{J}^{(2)}$, i.e. the heat flux for $f_{1}^{(2)}$, does not appear, the solubility conditions of the steady-state 
Boltzmann equation for $\phi_{1}^{(2)}$ lead to the heat flux being constant to second order. From eqs.(34) and (35), we also obtain an important relation between $(\nabla T)^{2}$ and $\nabla^{2} T$, namely

$$
\frac{(\nabla T)^{2}}{2 T}+\nabla^{2} T=0
$$

Owing to the relation (36), terms of $\nabla^{2} T$ can be replaced by terms of $(\nabla T)^{2}$.

\section{The second order}

We write down the results of the second-order coefficients $B_{k r}^{\mathrm{II}}$ of which $\phi_{1}^{(2)}$ is composed. We can determine the second-order coefficients $B_{0 r}^{\mathrm{II}}$ appearing in eq.(14) as

$$
B_{0 r}^{\mathrm{II}}=\frac{b_{0 r}}{\pi \sigma^{4} n^{2} T^{2}}(\nabla T)^{2} .
$$

Values for the constants $b_{0 r}$ are summarized in Table III. The calculation of $B_{0 r}^{\mathrm{II}}$ is shown in Appendix D. It must be emphasized that Burnett did not obtain the second-order coefficients $B_{0 r}^{\mathrm{II}}$ because he was only interested in the family of the coefficient $B_{20}^{\mathrm{II}}$ which is related to the pressure tensor $P_{i j}$. 15] The necessity of the second-order coefficients $B_{0 r}^{\mathrm{II}}$ for the calculation of some physical quantities will be discussed later. We have calculated $B_{0 r}^{\mathrm{II}}$ to 7th approximation, i.e. $B_{0 r}^{\mathrm{II}}$ for $r \leq 6$ in this paper.

The other second-order coefficients $B_{k r}^{\mathrm{II}}$ in eq.(16) can be written in the final form:

$$
B_{k r}^{\mathrm{II}}=\frac{\delta_{k, 2} b_{2 r}}{\pi \sigma^{4} n^{2} T^{2}}\left[2\left(\partial_{z} T\right)^{2}-\left(\partial_{x} T\right)^{2}-\left(\partial_{y} T\right)^{2}\right] .
$$

Values for the constants $b_{2 r}$ are summarized in Table III. The calculation of $B_{k r}^{\mathrm{II}}$ is explained in Appendix D, Our 4th approximation $B_{20}^{\mathrm{II}}$ deviates from Burnett's 4th approximation $B_{20}^{\mathrm{II}}$ by a factor 1.003. This deviation is considered to be due to errors in Burnett's calculation of the second and the third terms on the right-hand side of eq.(D16). Although Burnett obtained $B_{k r}^{\mathrm{II}}$ only to 4 th approximation, i.e. $B_{k r}^{\mathrm{II}}$ for $r \leq 3$, we have obtained $B_{k r}^{\mathrm{II}}$ to 7 th approximation, i.e. $B_{k r}^{\mathrm{II}}$ for $r \leq 6$ in the present paper. Owing to the properties of the spherical harmonic function[15], $B_{k r}^{(1) \mathrm{II}}$ can be obtained by replacing $2\left(\partial_{z} T\right)^{2}-\left(\partial_{x} T\right)^{2}-$ $\left(\partial_{y} T\right)^{2}$ in eq.(38) by $6 \partial_{z} T \partial_{x} T$ using an axis change; similarly, $C_{k r}^{(1) I I}$ can be obtained by replacing $2\left(\partial_{z} T\right)^{2}-\left(\partial_{x} T\right)^{2}-\left(\partial_{y} T\right)^{2}$ by $6 \partial_{z} T \partial_{y} T$, and $B_{k r}^{(2) I I}$ can be obtained by replacing $2\left(\partial_{z} T\right)^{2}-\left(\partial_{x} T\right)^{2}-\left(\partial_{y} T\right)^{2}$ in eq.(38) by $6\left[\left(\partial_{x} T\right)^{2}-\left(\partial_{y} T\right)^{2}\right]$ via an axis change; $C_{k r}^{(2) \mathrm{II}}$ by replacement by $12 \partial_{x} T \partial_{y} T$. Note that other terms, e.g. $B_{k r}^{(3) \mathrm{II}}$, do not appear because $p$ in $B_{k r}^{(p) I I}$ must be $k$ or less, from eq.(16). 
One can see that both of $B_{0 r}^{\mathrm{II}}$ and $B_{k r}^{\mathrm{II}}$ are of the order of $K^{2}$. As is mentioned in Appendix D. we have found the fact that $B_{k r}^{\mathrm{II}}$ for $k=4,6$ and 8 do not appear, which strongly suggests that $B_{k r}^{\mathrm{II}}$ for all $k$ greater than 2 do not appear. Thus, we expect $B_{k r}^{\mathrm{II}}=0$ for $k \neq 2$ although Burnett 15] had believed that they would appear. We have calculated the second-order coefficients now, so that we finally obtain $f_{1}^{(2)}$ by substituting the second-order coefficients obtained here into eqs.(14) and (16).

\section{The velocity distribution functions to second order}

The velocity distribution function for hard-core molecules which we have derived in @IIC 1 and IIC3 valid to second order is now applied to a nonequilibrium steady-state system under the temperature gradient along $x$-axis. It can be written as

$$
\begin{aligned}
f=f^{(0)}\{1 & -\frac{4 J_{x}}{5 b_{11} n \kappa T}\left(\frac{m}{2 \kappa T}\right)^{\frac{1}{2}} \sum_{r \geq 1} r ! b_{1 r} c_{x} \Gamma\left(r+\frac{5}{2}\right) S_{\frac{3}{2}}^{r}\left(\mathbf{c}^{2}\right) \\
& +\frac{4096 m J_{x}^{2}}{5625 b_{11}^{2} n^{2} \kappa^{3} T^{3}}\left[\sum_{r \geq 2} r ! b_{0 r} \Gamma\left(r+\frac{3}{2}\right) S_{\frac{1}{2}}^{r}\left(\mathbf{c}^{2}\right)\right. \\
& \left.\left.+\sum_{r \geq 0} r ! b_{2 r}\left(2 c_{x}^{2}-c_{y}^{2}-c_{z}^{2}\right) \Gamma\left(r+\frac{7}{2}\right) S_{\frac{5}{2}}^{r}\left(\mathbf{c}^{2}\right)\right]\right\}
\end{aligned}
$$

where the specific values for $b_{1 r}, b_{0 r}$ and $b_{2 r}$ are found in Tables 【1 II and III, respectively, and $J_{x}$ corresponds to the $x$ component of the heat flux in eq.(35). Note that we have changed $\mathbf{c}_{1}$ to $\mathbf{c}$. Figure 2 provides the explicit form of the second-order velocity distribution function of the steady-state Boltzmann equation $\phi^{(2)}(\mathbf{c})$ for hard-core molecules with 7 th approximation $b_{0 r}$ and $b_{2 r}$, scaled by $m J_{x}^{2} / n^{2} \kappa^{3} T^{3}$. Figure 2 shows that the scaled $\phi^{(2)}(\mathbf{c})$ for hard-core molecules is strained symmetrically.

In order to compare the macroscopic quantities for hard-core molecules with those for Maxwell molecules, we also adopt the velocity distribution function for Maxwell molecules derived by Schamberg [16] for our calculation of the macroscopic quantities. We apply Schamberg's velocity distribution function to a nonequilibrium steady-state system under the temperature gradient along $x$-axis, that is, we take out the differential coefficients with respect to time $t$ from Schamberg's velocity distribution function and also use the solubility condition (34) for Maxwell molecules. The precise velocity distribution function of the steady-state 
Boltzmann equation for Maxwell molecules to second order finally becomes 16]

$$
\begin{aligned}
f=f^{(0)}\{1 & -\frac{4 J_{x}}{5 n \kappa T}\left(\frac{m}{2 \kappa T}\right)^{\frac{1}{2}} b_{11} c_{x} \Gamma\left(\frac{7}{2}\right) S_{\frac{3}{2}}^{1}\left(\mathbf{c}^{2}\right)+\frac{4096 m J_{x}^{2}}{5625 n^{2} \kappa^{3} T^{3}}\left[\sum_{r=2,3} r ! b_{0 r} \Gamma\left(r+\frac{3}{2}\right) S_{\frac{1}{2}}^{r}\left(\mathbf{c}^{2}\right)\right. \\
& \left.\left.+\sum_{r=1,2} r ! b_{2 r}\left(2 c_{x}^{2}-c_{y}^{2}-c_{z}^{2}\right) \Gamma\left(r+\frac{7}{2}\right) S_{\frac{5}{2}}^{r}\left(\mathbf{c}^{2}\right)\right]\right\} .
\end{aligned}
$$

As can be seen from eqs.(39) and (40), the explicit form of the velocity distribution function for hard-core molecules becomes the sum of an infinite series of Sonine polynomials, while the precise form of the velocity distribution function for Maxwell molecules is the sum of five Sonine polynomials. Figure 3 gives the direct comparison of the scaled $\phi^{(2)} \mathrm{s}$ in eq. (39) with 4th, 5th, 6th and 7th approximation $b_{0 r} \mathrm{~s}$ and $b_{2 r} \mathrm{~s}$ with the scaled $\phi^{(2)}$ in eq.(40). It should be mentioned that, as Fig 3 shows, the scaled $\phi^{(2)}$ in eq.(39) has not yet converged to 4th approximation. Furthermore, in this paper, we adopt the precise expression of the corresponding velocity distribution function for the steady-state BGK equation, though we do not write down the explicit form of it, which can be reduced from the general form of the the Chapman-Enskog solution for the steady-state BGK equation to arbitrary order [26, 27, 28, 29, 30]. We mention that $\phi^{(1)}$ for the steady-state BGK equation is identical to that for the steady-state Boltzmann equation for Maxwell molecules appearing in eq.(40), while $\phi^{(2)} \mathrm{s}$ are different from each other.

\section{THERMODYNAMIC QUANTITIES}

We can introduce the general form of the heat flux as

$$
J_{x}=-\varpi T^{\varphi} \partial_{x} T
$$

where $\varphi$ indicates temperature dependence of the thermal conductivity and $\varpi$ is a constant that depends upon microscopic models. $J_{x}$ is constant from the solubility condition (34). For example, $\varphi$ is calculated as 1/2 for hard-core molecules and 1 for Maxwell molecules; $\varpi$ is determined as $75 b_{11} \kappa(\kappa / \pi m)^{1 / 2} / 64 \sigma^{2}$ with $b_{11} \simeq 1.025$ for hard-core molecules ( see eq.(35)) and $5 \kappa^{2}(2 m / G)^{1 / 2} / 4 A$ for Maxwell molecules [14], where $G$ is the constant of proportionality between the intermolecular force and the reciprocal fifth power of the distance, and $A$ is a number constant erroneously evaluated as 1.3682 by Maxwell 12, 13] and recalculated as 1.3700 by Chapman[43]. Note that $\varphi$ and $\varpi$ cannot be determined explicitly from the BGK 
equation. From eq.(41), the temperature profile $T(x)$ in the nonequilibrium steady state can be determined as

$$
\begin{aligned}
T(x) & =\left[T(0)^{\varphi+1}-(\varphi+1) \frac{J_{x}}{\varpi} x\right]^{\frac{1}{\varphi+1}} \\
& \simeq T(0)\left[1-\frac{J_{x}}{\varpi T(0)^{\varphi+1}} x-\frac{\varphi J_{x}^{2}}{2 \varpi^{2} T(0)^{2 \varphi+2}} x^{2}\right],
\end{aligned}
$$

to second order. The temperature profile $T(x)$ becomes nonlinear except for $\varphi=0$. This fact is in accord with a numerical result that a temperature profile becomes nonlinear as the heat flux becomes larger. 44 ]

Using eq.(21), the pressure tensor $P_{i j}$ in the nonequilibrium steady state can be obtained as

$$
P_{i j}=n \kappa T\left[\delta_{i j}+\lambda_{P}^{i j} \frac{m J_{x}^{2}}{n^{2} \kappa^{3} T^{3}}\right]
$$

with the unit tensor $\delta_{i j}$ and the tensor components $\lambda_{P}^{i j}$ given in Table IV. Note that the off-diagonal components of $\lambda_{P}^{i j}$ are zero and $\lambda_{P}^{y y}=\lambda_{P}^{z z}$ is satisfied. The values of $\lambda_{P}^{i j}$ for 7 th approximation $b_{1 r}, b_{0 r}$ and $b_{2 r}$ for hard-core molecules, i.e. 7 th approximation $\lambda_{P}^{i j}$ seems to be converged to three significant figures, as can be seen from Table IV] We find that $\lambda_{P}^{i j}$ for hard-core molecules differs from that for Maxwell molecules not only quantitatively but also qualitatively: $\lambda_{P}^{x x} \neq \lambda_{P}^{y y}=\lambda_{P}^{z z}$ for hard-core molecules in the nonequilibrium steady state, while $\lambda_{P}^{x x}=\lambda_{P}^{y y}=\lambda_{P}^{z z}=0$ for Maxwell molecules. For hard-core molecules, $P_{x x}$ becomes smaller than $P_{y y}$ and $P_{z z}$ regardless of the sign of $J_{x}$. It is also important that, since $n \kappa T$ is required to be uniform from the solubility conditions for $\phi^{(1)}$ in eq. (8) ), we find from eq.(42) that $P_{i j}$ in eq.(43) is uniform to second order in the steady state. Additionally, since the temperature profile $T(x)$ has already been given in eq.(42), we can determine the density profile $n(x)$ to second order for uniform $P_{i j}$. Note that the equation of state in the nonequilibrium steady state is not modified to first order, and that $\lambda_{P}^{i j}$ for the steady-state BGK equation is identical with that for Maxwell molecules.

Each component of the kinetic temperature in the nonequilibrium steady state, i.e. $T_{\mathrm{i}}$ for $\mathrm{i}=x, y$ and $z$ is calculated as

$$
\begin{aligned}
\frac{n \kappa T_{\mathrm{i}}}{2} & \equiv\left(\frac{2 \kappa T}{m}\right)^{\frac{5}{2}} \int_{-\infty}^{\infty} \mathrm{d} \mathbf{c} \frac{m c_{\mathrm{i}}^{2}}{2} f \\
& =\frac{n \kappa T}{2}\left[1+\lambda_{T_{\mathrm{i}}} \frac{m J_{x}^{2}}{n^{2} \kappa^{3} T^{3}}\right]
\end{aligned}
$$


for $\mathrm{i}=x, y$ and $z$. Values for the constants $\lambda_{T_{\mathrm{i}}}$ for $\mathrm{i}=x, y$ and $z$ are given in Table IV. Note the relation $\lambda_{T_{y}}=\lambda_{T_{z}}$. $\lambda_{T_{\mathrm{i}}}$ with 7th approximation $b_{1 r}, b_{0 r}$ and $b_{2 r}$ for hard-core molecules, i.e. 7th approximation $\lambda_{T_{\mathrm{i}}}$ seems to converge to three significant figures, according to the results reported in Table IV] Since $n \kappa T$ and $J_{x}$ are uniform, we see that the correction term of $T_{\mathrm{i}}$ in eq.(44) is independent of $x$. The value of $\lambda_{T_{\mathrm{i}}}$ for hard-core molecules is seen to be qualitatively different from that for Maxwell molecules: $\lambda_{T_{x}} \neq \lambda_{T_{y}}=\lambda_{T_{z}}$ for hard-core molecules in the nonequilibrium steady-state, while $\lambda_{T_{x}}=\lambda_{T_{y}}=\lambda_{T_{z}}=0$ for Maxwell molecules in that state. For hard-core molecules, $T_{x}$ becomes smaller than $T_{y}$ and $T_{z}$ regardless of the sign of $J_{x}$, which means that the motion of hard-core molecules along the heat flux becomes dull. We note that $T_{\mathrm{i}}$ for the steady-state BGK equation is identical with that for Maxwell molecules, and that, to first order, $T_{\mathrm{i}}$ for hard-core molecules is isotropic.

The Shannon entropy in the nonequilibrium steady state $S$ is defined via

$$
\begin{aligned}
S & \equiv-\kappa\left(\frac{2 \kappa T}{m}\right)^{\frac{3}{2}} \int_{-\infty}^{\infty} \mathrm{d} \mathbf{c} f \log f \\
& =-n \kappa \log \left[n\left(\frac{m}{2 \pi \kappa T}\right)^{\frac{3}{2}}\right]+\frac{3}{2} n \kappa+\lambda_{S} \frac{m J_{x}^{2}}{n \kappa^{2} T^{3}} .
\end{aligned}
$$

Values for the constant $\lambda_{S}$ are given in Table IV $\lambda_{S}$ for 7 th approximation $b_{1 r}, b_{0 r}$ and $b_{2 r}$ for hard-core molecules, i.e. 7th approximation $\lambda_{S}$ seems to converge to four significant figures, see Table IV. It is found that $\lambda_{S}$ for hard-core molecules is close to that for Maxwell molecules, while the latter is identical with that for the steady-state BGK equation. Note that the Shannon entropy in the nonequilibrium steady state is not modified to first order.

\section{DISCUSSION}

It is noteworthy that all macroscopic quantities now become able to be calculated directly from the explicit velocity distribution functions of the steady-state Boltzmann equation to second order. Actually, as in Appendix E we examine the nonequilibrium steady-state thermodynamics(SST) proposed by Oono and Paniconi [45], and extended by Sasa and Tasaki [46] by using the velocity distribution function to second order for hard-core molecules we have derived in $₫$ III and that for Maxwell molecules. As a result, our results do not support SST. We mention that the full information of the second-order coefficients $B_{0 r}^{\mathrm{II}}$ and $B_{k r}^{\mathrm{II}}$ is necessary for the calculation of some physical quantities in the test of SST. (see Appendix E) Furthermore, as indicated in the introduction, the other important application of the 
explicit second-order velocity distribution function for hard-core molecules is to evaluate the nonequilibrium effect on rate of chemical reaction of gases. The line-of-centers model[47] which is accepted as a standard one requires the full form of the second-order velocity distribution function for hard-core molecules 40]. Our result enables us to calculate the reaction rate. [41]

We have found that there are qualitative differences between hard-core molecules and Maxwell molecules in the nonequilibrium steady state: second-order corrections appear for hard-core molecules in the pressure tensor $P_{i j}$ and the kinetic temperature $T_{\mathrm{i}}$, while no correction to these quantities appears for Maxwell molecules, as Table IV shows. It should be noted that the qualitative differences between hard-core molecules and Maxwell molecules still appear no matter which boundary condition is adopted, that is, the isotropy and the anisotropy of the pressure tensor in eq.(43) and the kinetic temperature in eq.(44) are not affected by any kinds of boundary conditions. It is conjectured that these differences are attributed to the special nature of Maxwell molecules: $\Delta_{k r}$ for Maxwell molecules, i.e. $\Delta_{k r}^{\mathrm{M}}$ is independent of the magnitude of the relative velocity $g$, because $F_{k r}^{0}(\chi)$ is independent of $g$. In general, $\Delta_{k r}$ for molecules which interact with each other by a central force depends on $g$, because $F_{k r}^{\mu}(\chi)$ generally depends on $g$. Therefore, it may be suggested that molecules which interact via a central potential still have the qualitative differences from Maxwell molecules: second-order corrections in pressure tensor $P_{i j}$ and kinetic temperature $T_{\mathrm{i}}$ may appear also for such molecules as well as hard-core molecules.

It is also found that the pressure tensor $P_{i j}$ and the kinetic temperature $T_{\mathrm{i}}$ for the steadystate BGK equation are qualitatively different from those for the steady-state Boltzmann equation for hard-core molecules but agree with those for the steady-state Boltzmann equation for Maxwell molecules, as illustrated in Table IV. Since the Chapman-Enskog solution of the steady-state BGK equation is asymptotically correct 26, 27, 28, 29, 30], we may conclude that the steady-state BGK equation does not capture the essence of hard-core molecules, but captures that of Maxwell-type molecules. This conclusion indicates the possibility that even the exact solution of the steady-state BGK equation can be applied only to Maxwell-type molecules.

Finally, we consider the possibility of the existence of a universal velocity distribution function in the nonequilibrium steady state. When we express the velocity distribution function for hard-core molecules to second order using the heat flux $J_{x}$ as in eq.(39), it be- 
comes independent of the diameter $\sigma$ of the hard-core molecules. However, the explicit form of the velocity distribution function for hard-core molecules (39) definitely differs from the precise form of the velocity distribution function for Maxwell molecules (40) as Fig [3] shows. Actually, as mentioned above, we have shown that the results calculated from the former are qualitatively different from those calculated from the latter. These results indicate that the characteristics of microscopic models appear in a nonequilibrium steady state solution and affect even qualitatively the macroscopic quantities of a system in that nonequilibrium steady state. It can be concluded that a universal velocity distribution function does not exist in the nonequilibrium steady state even if the velocity distribution function is expressed only in terms of macroscopic quantities. [48]

\section{CONCLUSION}

The velocity distribution function of the steady-state Boltzmann equation for hard-core molecules subject to a temperature gradient has been derived explicitly to second order in the density and the temperature gradient as shown explicitly in eq.(39) and Fig[2. In the nonequilibrium steady-state system, qualitative differences between hard-core molecules and Maxwell molecules are found in the pressure tensor (43) and the kinetic temperature (44): it appears that Maxwell molecules do not possess the characteristics of other models of molecules which interact with each other by central forces in the nonequilibrium steadystate system. Additionally, we have found that the steady-state BGK equation belongs to the same universality class as Maxwell molecules, and that it does not capture the essence of hard-core molecules. We finally conclude that a universal velocity distribution function does not seem to exist, as Fig 3 explicitly shows, even when the velocity distribution function is expressed only in terms of macroscopic quantities.

\section{Acknowledgments}

We would like to express our sincere gratitude to S. Sasa who has made us aware of the significance of understanding nonequilibrium steady-state phenomena. This research was essentially inspired by him. We thank H. Tasaki who has always made the crucial, interesting and cheerful discussions with us and who has encouraged us to carry out these calculations. 
The authors also appreciate Ooshida. T, A. Yoshimori, M. Sano, J. Wakou, K. Sato, H. Kuninaka, T. Mizuguti, T. Chawanya, S. Takesue and H. Tomita for fruitful discussions and useful comments. We thank A. Santos to provide helpful and important papers for us. This study has been supported partially by the Hosokawa Powder Technology Foundation, the Inamori Foundation and Grant-in-Aid for Scientific Research (No. 13308021).

\section{APPENDIX A: THE CALCULATION OF $\Omega_{k r}$}

From the definition of $Q_{k r}, \Omega_{k r}$ can be calculated using the mathematical properties of the spherical harmonic functions and the Sonine polynomials. 15, 16] One can calculate $D_{k, r}$, $E_{k, r}$ and $G_{k, r}$ defined as

$$
D_{k, r} \equiv \frac{1}{n}<c_{1 x} Q_{k r}>_{a v}, \quad E_{k, r} \equiv \frac{1}{n}<c_{1 y} Q_{k r}>_{a v} \quad \text { and } \quad G_{k, r} \equiv \frac{1}{n}<c_{1 z} Q_{k r}>_{a v} .
$$

The results can be written as

$$
D_{k, r}=\frac{1}{2 k+3}\left[\left(k+r+\frac{3}{2}\right) B_{k+1, r}^{(1)}-B_{k+1, r-1}^{(1)}\right]-\frac{1}{2 k-1}\left[B_{k-1, r}^{(1)}-(r+1) B_{k-1, r+1}^{(1)}\right],
$$

and

$$
E_{k, r}=\frac{1}{2 k+3}\left[\left(k+r+\frac{3}{2}\right) C_{k+1, r}^{(1)}-C_{k+1, r-1}^{(1)}\right]-\frac{1}{2 k-1}\left[C_{k-1, r}^{(1)}-(r+1) C_{k-1, r+1}^{(1)}\right],
$$

and

$$
G_{k, r}=\frac{k+1}{2 k+3}\left[\left(k+r+\frac{3}{2}\right) B_{k+1, r}-B_{k+1, r-1}\right]-\frac{k}{2 k-1}\left[B_{k-1, r}-(r+1) B_{k-1, r+1}\right] .
$$

One can also obtain

$$
<c_{1 x} \partial_{x} Q_{k r}>_{a v}=-n \frac{\partial_{x} T}{T}\left[\left(r+\frac{k}{2}\right) D_{k, r}-D_{k, r-1}\right] .
$$

Similarly $<c_{1 y} \partial_{y} Q_{k r}>_{a v}$ and $<c_{1 z} \partial_{z} Q_{k r}>_{a v}$ are obtained by replacing the differential coefficients with respect to $x$ by the corresponding differential coefficients with respect to $y$ and $z$, the $D_{k, r}$ 's by the corresponding $E_{k, r}$ 's and $G_{k, r}$ 's, respectively. Substituting these results into eq.(24), $\Omega_{k r}$ finally becomes eq.(26) . 


\section{APPENDIX B: THE DETAILS OF $F_{k r}^{1}(\chi)$}

The details of $F_{k r}^{1}(\chi)$ are as follows. Substituting the general forms of $f_{1}, f_{2}$ in eq.(14) and $Q_{k r}^{\prime}$ in eq.(22) into $F_{k r}^{1}(\chi)$ in eq.(29), $F_{k r}^{1}(\chi)$ can be written as 15$]$

$$
F_{k r}^{1}(\chi)=\sum_{n_{1}, n_{2}, k_{1}, k_{2}, k_{1} \geq p_{1} \geq 0, k_{2} \geq p_{2} \geq 0} W_{k, k_{1}, k_{2}}^{n_{1}, n_{2}, p_{1}, p_{2}} \Xi_{k, k_{1}, k_{2}}^{r, n_{1}, n_{2}, p_{1}, p_{2}}(\chi)
$$

where the summation with respect to $p_{1}$ and $p_{2}$ is performed from 0 to $k_{1}$ and $k_{2}$, respectively as seen in eq.(16). Here $\Xi_{k, k_{1}, k_{2}}^{r, n_{1}, n_{2}, p_{1}, p_{2}}(\chi)$ is the characteristic integral defined as

$$
\begin{aligned}
\Xi_{k, k_{1}, k_{2}}^{r, n_{1}, n_{2}, p_{1}, p_{2}}(\chi) & \equiv \Gamma\left(k_{1}+n_{1}+\frac{3}{2}\right) \Gamma\left(k_{2}+n_{2}+\frac{3}{2}\right)\left(\frac{2 \kappa T}{m}\right)^{3} \iiint Y_{k}\left(\mathbf{c}_{1}^{\prime}\right) Y_{k_{1}}^{\left(p_{1}\right)}\left(\mathbf{c}_{1}\right) Y_{k_{2}}^{\left(p_{2}\right)}\left(\mathbf{c}_{2}\right) \\
& \times \exp \left[-\left(c_{1}^{2}+c_{2}^{2}\right)\right] S_{k+\frac{1}{2}}^{r}\left(\mathbf{c}_{1}^{\prime 2}\right) S_{k_{1}+\frac{1}{2}}^{n_{1}}\left(\mathbf{c}_{1}^{2}\right) S_{k_{2}+\frac{1}{2}}^{n_{2}}\left(\mathbf{c}_{2}^{2}\right) g \mathrm{~d} \epsilon \mathrm{d} \mathbf{c}_{2} \mathrm{~d} \mathbf{c}_{1} .
\end{aligned}
$$

Note that the integrals containing products like $Y_{k_{1}}^{\left(p_{1}\right)}\left(\mathbf{c}_{1}\right) Z_{k_{2}}^{\left(p_{2}\right)}\left(\mathbf{c}_{2}\right)$ are zero, owing to the orthogonality properties of the spherical harmonic functions, while those containing $Z_{k_{1}}^{\left(p_{1}\right)}\left(\mathbf{c}_{1}\right) Z_{k_{2}}^{\left(p_{2}\right)}\left(\mathbf{c}_{2}\right)$ are identical with the corresponding integrals $\Xi_{k, k_{1}, k_{2}}^{r, n_{1}, n_{2}, p_{1}, p_{2}}(\chi)$. The factor $W_{k, k_{1}, k_{2}}^{n_{1}, n_{2}, p_{1}, p_{2}}$ in eq. (B1) is defined as

$$
\begin{array}{r}
W_{k, k_{1}, k_{2}}^{n_{1}, n_{2}, p_{1}, p_{2}} \equiv n^{2}\left(\frac{m}{2 \pi \kappa T}\right)^{3}\left(\frac{m}{2 \kappa T}\right)^{\frac{k+k_{1}+k_{2}}{2}} n_{1} ! n_{2} !\left(k+\frac{1}{2}\right) \sqrt{\pi} \\
\Psi_{k_{1}, k_{2}}^{p_{1}, p_{2}}\left(B_{k_{1} n_{1}}^{\left(p_{1}\right)} B_{k_{2} n_{2}}^{\left(p_{2}\right)}+C_{k_{1} n_{1}}^{\left(p_{1}\right)} C_{k_{2} n_{2}}^{\left(p_{2}\right)}\right)
\end{array}
$$

which is obtained from the prefactors and the coefficients in the general form of $f_{1}, f_{2}$ in eq.(14) and $Q_{k r}^{\prime}$ in eq.(22). Note that $C_{k_{1} n_{1}}^{\left(p_{1}\right)} C_{k_{2} n_{2}}^{\left(p_{2}\right)}$ appear from the integrals containing $Z_{k_{1}}^{\left(p_{1}\right)}\left(\mathbf{c}_{1}\right) Z_{k_{2}}^{\left(p_{2}\right)}\left(\mathbf{c}_{2}\right)$, and that $B_{k r}^{(0)}=B_{k r}$ and $C_{k r}^{(0)}=0$ from eqs.(16), (17), (18) and (19). The constant $\Psi_{k_{1}, k_{2}}^{p_{1}, p_{2}}$ is defined as

$$
\Psi_{k_{1}, k_{2}}^{p_{1}, p_{2}}=1 \quad \text { for } \quad p_{1}=p_{2}=0
$$

and

$$
\Psi_{k_{1}, k_{2}}^{p_{1}, p_{2}}=\frac{4\left(k_{1}-p_{1}\right) !\left(k_{2}-p_{1}\right) !}{\left(k_{1}+p_{1}\right) !\left(k_{2}+p_{1}\right) !} \quad \text { for } \quad p_{1}=p_{2} \neq 0 .
$$

Here $\Psi_{k_{1}, k_{2}}^{p_{1}, p_{2}}$ for $p_{1} \neq p_{2}$ is not necessary for our calculation. 15, 49] It is found that we need only to evaluate the characteristic integral $\Xi_{k, k_{1}, k_{2}}^{r, n_{1}, n_{2}, p_{1}, p_{1}}(\chi)$ in order to calculate $F_{k r}^{1}(\chi)$. Our calculation of $\Xi_{k, k_{1}, k_{2}}^{r, n_{1}, n_{2}, p_{1}, p_{1}}(\chi)$ is written in ref. [49]. Our calculation has been performed mainly based on Burnett's method[15]. We have, however, made some modifications on his 
method, which make the calculation of $\Xi_{k, k_{1}, k_{2}}^{r, n_{1}, n_{2}, p_{1}, p_{1}}(\chi)$ much easier. 49] We emphasize that our calculation could not be carried out completely if we did not make the modifications on Burnett's method.

Once the characteristic integral $\Xi_{k, k_{1}, k_{2}}^{r, n_{1}, n_{2}, p_{1}, p_{1}}(\chi)$ has been derived, $F_{k r}^{1}(\chi)$ is now calculated from eq.(B1) with $W_{k, k_{1}, k_{2}}^{n_{1}, n_{2}, p_{1}, p_{1}}$ in eq. (BB3). Note that the coefficient term $B_{k_{1} n_{1}}^{\left(p_{1}\right)} B_{k_{2} n_{2}}^{\left(p_{2}\right)}+$ $C_{k_{1} n_{1}}^{\left(p_{1}\right)} C_{k_{2} n_{2}}^{\left(p_{2}\right)}$ in $W_{k, k_{1}, k_{2}}^{n_{1}, n_{2}, p_{1}, p_{1}}$ can be determined for each order when the suffices $k_{1}, k_{2}, n_{1}, n_{2}$, $p_{1}$ and $p_{2}$ are specified.

\section{APPENDIX C: THE CALCULATION OF THE FIRST ORDER COEFFICIENTS} $B_{k r}^{\mathrm{I}}$

Let us explain how to obtain the first-order coefficients, that is, how to solve the integral equation (51). To begin with, we calculate $\Omega_{k r}^{\mathrm{H}}$ in eq.(26) to first order; $\Omega_{k r}^{\mathrm{H}}$ for first order corresponds to the right-hand side of eq.(5). For first order, $\Omega_{k r}^{\mathrm{H}}$ in eq.(26) can be calculated by substituting $B_{00}=1$ into the expressions of $D_{k, r}, E_{k, r}$ and $G_{k, r}$ in eqs. (A2), (A3) and (A4): the coefficient $B_{00}=1$ corresponds to $f_{1}=f_{1}^{(0)}$, and higher-order terms do not appear in $\Omega_{k r}^{\mathrm{H}}$ for first order. $\Omega_{k r}^{\mathrm{H}}$ for first order finally becomes

$$
\Omega_{1 r}^{\mathrm{H}}=-\frac{n}{T}\left(\frac{m}{2 \kappa T}\right)^{-\frac{1}{2}} \frac{\partial T}{\partial z} \delta_{1, r}
$$

Now $\Omega_{k r}^{\mathrm{H}}$ for first order is found to vanish unless $k=1$, so that we need calculate only $\Delta_{1, r}^{\mathrm{H}}$ for first order; as was mentioned in the end of \&II we do not need to consider the case in which the right-hand side of eq.(15) becomes zero. [2] To derive $\Delta_{1, r}^{\mathrm{H}}$ in eq.(28) for first order, we must calculate both $W_{1, k_{1}, k_{2}}^{n_{1}, n_{2}, p_{1}, p_{1}}$ and $\Xi_{1, k_{1}, k_{2}}^{r, n_{1}, n_{2}, p_{1}, p_{1}}$ in $F_{1, r}^{1}(\chi)$ of eq. (B1) for first order as was shown in the previous subsection. The result for $\Delta_{1, r}^{\mathrm{H}}$ to first order can be written finally in the form

$$
\Delta_{1 r}^{\mathrm{H}}=B_{00} \sum_{n_{1}} B_{1 n_{1}}^{\mathrm{I}} M_{1,1,0}^{r, n_{1}, 0,0,0}
$$

where the set of the coefficients $B_{1 n_{1}}^{\mathrm{I}} B_{00}$ is obtained from $W_{1,1,0}^{n_{1}, 0,0,0}$ in eq.(B33). $f_{1}$ in eq.(29) contains only $B_{00}=1$ and the first-order coefficients, i.e. the family of $B_{k_{1} n_{1}}^{\mathrm{I}}$ to first order; $f_{2}$ in eq.(29) also contains $B_{00}=1$ and the family of $B_{k_{2} n_{2}}^{\mathrm{I}}$ to first order. Thus, we obtain only the term $B_{1 n_{1}}^{\mathrm{I}} B_{00}$ from $W_{1,1,0}^{n_{1}, 0,0,0}$ to first order using the fact that $F_{k r}^{1}(\chi)=0$ unless 
$k=\left|k_{1}-k_{2}\right|+2 q$. Note that it is sufficient to consider only the case for $k_{1} \geq k_{2}$ as is explained in refs. [15, 49], and that we set $q=0$. The matrix $M_{1,1,0}^{r, n_{1}, 0,0,0}$ is thus obtained

$$
\begin{aligned}
M_{1,1,0}^{r, n_{1}, 0,0,0} & =\frac{3 n^{2} \sigma^{2} m^{4} n_{1} !}{64 \pi^{\frac{5}{2}} \kappa^{4} T^{4}} \\
& \times \int_{0}^{\pi}\left[\Xi_{1,1,0}^{r, n_{1}, 0,0,0}(\chi)-\Xi_{1,1,0}^{r, n_{1}, 0,0,0}(0)\right] \sin \frac{\chi}{2} \cos \frac{\chi}{2} \mathrm{~d} \chi
\end{aligned}
$$

using eqs.(28), (B1) and (B3).

For $k=1$, eq.(32) gives simultaneous equations determining the first-order coefficients $B_{1 n_{1}}^{\mathrm{I}}$, i.e.

$$
\Omega_{1 r}^{\mathrm{H}}=\sum_{n_{1} \geq 1} B_{1 n_{1}}^{\mathrm{I}} M_{1,1,0}^{r, n_{1}, 0,0,0}
$$

from eqs. (C1) and (C2). Note that we need only to obtain the first-order coefficients $B_{1 n_{1}}^{\mathrm{I}}$ for $n_{1} \geq 1$, because $B_{10}=0$ from eq.(20). We have calculated the matrix $M_{1,1,0}^{r, n_{1}, 0,0,0}$ for $1 \leq r \leq 7$ and $1 \leq n_{1} \leq 7$ from eq.(C3), and we have confirmed that $M_{1,1,0}^{0, n_{1}, 0,0,0}$ for $1 \leq n_{1} \leq 7$ calculated from eq. (C3) vanishes. Our result for $M_{1,1,0}^{r, n_{1}, 0,0,0}$ for $1 \leq r \leq 7$ and $1 \leq n_{1} \leq 7$ is given in ref.[42]. At last, we can determine the first-order coefficients $B_{1 n_{1}}^{\mathrm{I}}$ by solving the simultaneous equations (C4), that is, $B_{1 n_{1}}^{\mathrm{I}}$ can be obtained as

$$
B_{1 n_{1}}^{\mathrm{I}}=\sum_{r \geq 1} \Omega_{1 r}^{\mathrm{H}}\left(M_{1,1,0}^{r, n_{1}, 0,0,0}\right)^{-1}
$$

where $X^{-1}$ represents the inverse matrix of a matrix $X$. Note that we have confirmed, using eqs.(C1) and (C3), that both sides of eq. (C4) for $r=0$ vanish, so that we need only calculate both sides of eq.(C4) for $r \geq 1$. Finally, the results of the first-order coefficients $B_{k_{1} n_{1}}^{\mathrm{I}}$, i.e. $B_{k r}^{\mathrm{I}}$ in eq.(16) can be calculated as in eq.(133).

\section{APPENDIX D: THE CALCULATION OF THE SECOND ORDER COEFFI-} CIENTS $B_{k r}^{\mathrm{II}}$

Let us explain how to obtain the second-order coefficients, that is, how to solve the integral equation (66). The coefficients of first order, i.e. the family of $B_{k r}^{\mathrm{I}}$, have been obtained as given in eq.(133), so that we can employ them to determine the second-order coefficients, i.e. the family of $B_{k r}^{\mathrm{II}}$.

To begin with, we calculate $\Omega_{k r}^{\mathrm{H}}$ in eq.(26) for second order; $\Omega_{k r}^{\mathrm{H}}$ for second order corresponds to the first term on the right-hand side of eq.(66). For second order, $\Omega_{k r}^{\mathrm{H}}$ in eq.(26) 
can be calculated by substituting the family of $B_{k r}^{\mathrm{I}}$ into the expressions of $D_{k, r}, E_{k, r}$ and $G_{k, r}$ in eqs. (A22), (A3) and (A4); other terms do not appear in $\Omega_{k r}^{\mathrm{H}}$ for second order. The results of the tedious calculation of $\Omega_{k r}^{\mathrm{H}}$ to second order finally become as follows. For $k=0$, $\Omega_{0 r}^{\mathrm{H}}$ becomes

$$
\Omega_{0 r}^{\mathrm{H}}=0
$$

for $r=0$ and 1 ,

$$
\Omega_{02}^{\mathrm{H}}=\frac{35}{32} \frac{1}{\sqrt{2 \pi} \sigma^{2} T^{2}}\left(\frac{m}{2 \kappa T}\right)^{-\frac{1}{2}}(\nabla T)^{2}\left(b_{12}-b_{11}\right),
$$

for $r=2$, and

$$
\Omega_{0 r}^{\mathrm{H}}=\frac{5}{16} \frac{1}{\sqrt{2 \pi} \sigma^{2} T^{2}}\left(\frac{m}{2 \kappa T}\right)^{-\frac{1}{2}}(\nabla T)^{2}\left[\left(r^{2}+\frac{r}{2}-\frac{3}{2}\right) b_{1 r}-\left(2 r-\frac{1}{2}\right) b_{1, r-1}+b_{1, r-2}\right],
$$

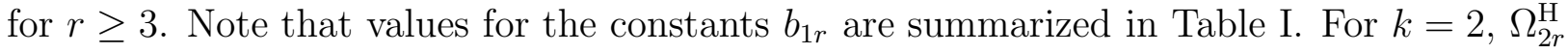
becomes

$$
\Omega_{20}^{\mathrm{H}}=0,
$$

and

$$
\Omega_{21}^{\mathrm{H}}=-\frac{5}{8} \frac{1}{\sqrt{2 \pi} \sigma^{2} T^{2}}\left(\frac{m}{2 \kappa T}\right)^{-\frac{1}{2}}\left[2\left(\partial_{z} T\right)^{2}-\left(\partial_{x} T\right)^{2}-\left(\partial_{y} T\right)^{2}\right]\left(b_{12}-b_{11}\right),
$$

and

$$
\begin{aligned}
\Omega_{2 r}^{\mathrm{H}} & =\frac{5}{16} \frac{1}{\sqrt{2 \pi} \sigma^{2} T^{2}}\left(\frac{m}{2 \kappa T}\right)^{-\frac{1}{2}}\left[2\left(\partial_{z} T\right)^{2}-\left(\partial_{x} T\right)^{2}-\left(\partial_{y} T\right)^{2}\right] \\
& \times\left[-r(r+1) b_{1, r+1}+2 r b_{1 r}-b_{1, r-1}\right]
\end{aligned}
$$

for $r \geq 2$. For $k=1$ and $k \geq 3$, we find that $\Omega_{k r}^{\mathrm{H}}$ becomes

$$
\Omega_{k r}^{\mathrm{H}}=0,
$$

for any value of $r$.

Next let us calculate $\Delta_{k r}^{\mathrm{H}}$ in eq.(28) for second order. In order to derive $\Delta_{k r}^{\mathrm{H}}$ for second order, we have to calculate $W_{k, k_{1}, k_{2}}^{n_{1}, n_{2}, p_{1}, p_{1}}$ and $\Xi_{k, k_{1}, k_{2}}^{r, n_{1}, n_{2}, p_{1}, p_{1}}$ in $F_{k r}^{1}(\chi)$ of eq. (B1) to second order, as was shown in Appendix $\mathrm{B}$. For even $k, \Delta_{k r}^{\mathrm{H}}$ to second order results in the general form:

$$
\begin{aligned}
\Delta_{k r}^{\mathrm{H}} & =B_{00} \sum_{n_{1}, 0 \leq q \leq \frac{k}{2}} B_{k-2 q, n_{1}}^{\mathrm{II}} M_{k, k-2 q, 0}^{r, n_{1}, 0,0,0}+\sum_{n_{1}, n_{2}} B_{1 n_{1}}^{\mathrm{I}} B_{1 n_{2}}^{\mathrm{I}} M_{k, 1,1}^{r, n_{1}, n_{2}, 0,0} \\
& +\sum_{n_{1}, n_{2}}\left[B_{1 n_{1}}^{(1) \mathrm{I}} B_{1 n_{2}}^{(1) \mathrm{I}}+C_{1 n_{1}}^{(1) \mathrm{I}} C_{1 n_{2}}^{(1) \mathrm{I}}\right] M_{k, 1,1}^{r, n_{1}, n_{2}, 1,1}
\end{aligned}
$$


Here the set of the coefficients $B_{k-2 q, n_{1}}^{\mathrm{II}} B_{00}$ can be obtained from $W_{k, k-2 q, 0}^{n_{1}, 0,0,0}$ in eq. (B33). The set of the coefficients $B_{1 n_{1}}^{\mathrm{I}} B_{1 n_{2}}^{\mathrm{I}}$ is obtained from $W_{k, 1,1}^{n_{1}, n_{2}, 0,0}$ in eq.(B3); $B_{1 n_{1}}^{\mathrm{I}}$ from $f_{1}$ and $B_{1 n_{2}}^{\mathrm{I}}$ from $f_{2}$ are the first-order coefficients obtained in eq.(133), so that $B_{1 n_{1}}^{\mathrm{I}} B_{1 n_{2}}^{\mathrm{I}}$ is second order. Similarly, the set of the terms $B_{1 n_{1}}^{(1) \mathrm{I}} B_{1 n_{2}}^{(1) \mathrm{I}}+C_{1 n_{1}}^{(1) \mathrm{I}} C_{1 n_{2}}^{(1) \mathrm{I}}$ is obtained from $W_{k, 1,1}^{n_{1}, n_{2}, 1,1}$ in eq.(B33); $B_{1 n_{1}}^{(1) \mathrm{I}}, C_{1 n_{1}}^{(1) \mathrm{I}}$ from $f_{1}$ and $B_{1 n_{2}}^{(1) \mathrm{I}}, C_{1 n_{2}}^{(1) \mathrm{I}}$ from $f_{2}$ are the first-order coefficients obtained in IIIC1, so that $B_{1 n_{1}}^{(1) \mathrm{I}} B_{1 n_{2}}^{(1) \mathrm{I}}$ and $C_{1 n_{1}}^{(1) \mathrm{I}} C_{1 n_{2}}^{(1) \mathrm{I}}$ are also second order. To second order, $f_{1}$ of eq.(29) contains only $B_{00}=1$, the family of $B_{k_{1} n_{1}}^{\mathrm{I}}$ obtained as eq.(33) and the family of $B_{k_{1} n_{1}}^{\mathrm{II}}$ to be determined here; $f_{2}$ of eq.(29) also contains only $B_{00}=1$, the family of $B_{k_{2} n_{2}}^{\mathrm{I}}$ and the family of $B_{k_{2} n_{2}}^{\mathrm{II}}$ to second order. Thus, we can only obtain the sets of the terms in eq. (D8) for second order by using the fact that $F_{k r}^{1}(\chi)=0$ unless $k=\left|k_{1}-k_{2}\right|+2 q$ : the second and the third terms on the right-hand side of eq.(D8) do not appear for odd $k$. Note that it is sufficient to consider only the case for $k_{1} \geq k_{2}$, as is explained in refs. 15, 49], and that $B_{1 n_{1}}^{(1) \mathrm{I}} C_{1 n_{2}}^{(1) \mathrm{I}}$ or $C_{1 n_{1}}^{(1) \mathrm{I}} B_{1 n_{2}}^{(1) \mathrm{I}}$ does not appear owing to the orthogonality properties of the spherical harmonic functions. (see Appendix $(\mathrm{B})$ The matrix $M_{k, k-2 q, 0}^{r, n_{1}, 0,0,0}$ in eq. (D8) is thus obtained as

$$
\begin{aligned}
M_{k, k-2 q, 0}^{r, n_{1}, 0,0,0} & =\frac{n^{2} \sigma^{2}}{2}\left(\frac{m}{2 \pi \kappa T}\right)^{3}\left(\frac{m}{2 \kappa T}\right)^{k-q} n_{1} !\left(k+\frac{1}{2}\right) \sqrt{\pi} \\
& \times \int_{0}^{\pi}\left[\Xi_{k, k-2 q, 0}^{r, n_{1}, 0,0,0}(\chi)-\Xi_{k, k-2 q, 0}^{r, n_{1}, 0,0,0}(0)\right] \sin \frac{\chi}{2} \cos \frac{\chi}{2} \mathrm{~d} \chi
\end{aligned}
$$

using eqs.(28), (B1) and (B3). Similarly, the matrices $M_{k, 1,1}^{r, n_{1}, n_{2}, 0,0}$ and $M_{k, 1,1}^{r, n_{1}, n_{2}, 1,1}$ in eq.(D8) are derived as

$$
\begin{aligned}
M_{k, 1,1}^{r, n_{1}, n_{2}, 0,0} & =\frac{n^{2} \sigma^{2}}{2}\left(\frac{m}{2 \pi \kappa T}\right)^{3}\left(\frac{m}{2 \kappa T}\right)^{\frac{k}{2}+1} n_{1} ! n_{2} !\left(k+\frac{1}{2}\right) \sqrt{\pi} \\
& \times \int_{0}^{\pi}\left[\Xi_{k, 1,1}^{r, n_{1}, n_{2}, 0,0}(\chi)-\Xi_{k, 1,1}^{r, n_{1}, n_{2}, 0,0}(0)\right] \sin \frac{\chi}{2} \cos \frac{\chi}{2} \mathrm{~d} \chi
\end{aligned}
$$

and

$$
\begin{aligned}
M_{k, 1,1}^{r, n_{1}, n_{2}, 1,1} & =\frac{n^{2} \sigma^{2}}{2}\left(\frac{m}{2 \pi \kappa T}\right)^{3}\left(\frac{m}{2 \kappa T}\right)^{\frac{k}{2}+1} n_{1} ! n_{2} !\left(k+\frac{1}{2}\right) \sqrt{\pi} \\
& \times \int_{0}^{\pi}\left[\Xi_{k, 1,1}^{r, n_{1}, n_{2}, 1,1}(\chi)-\Xi_{k, 1,1}^{r, n_{1}, n_{2}, 1,1}(0)\right] \sin \frac{\chi}{2} \cos \frac{\chi}{2} \mathrm{~d} \chi
\end{aligned}
$$

respectively.

For even $k$, eq.(32) finally leads to simultaneous equations to determine the second-order 
coefficients $B_{k-2 q, n_{1}}^{\mathrm{II}}$ :

$$
\begin{aligned}
\Omega_{k r}^{\mathrm{H}} & =\sum_{n_{1}, 0 \leq q \leq \frac{k}{2}} B_{k-2 q, n_{1}}^{\mathrm{II}} M_{k, k-2 q, 0}^{r, n_{1}, 0,0,0}+\sum_{n_{1}, n_{2}} B_{1 n_{1}}^{\mathrm{I}} B_{1 n_{2}}^{\mathrm{I}} M_{k, 1,1}^{r, n_{1}, n_{2}, 0,0} \\
& +\sum_{n_{1}, n_{2}}\left[B_{1 n_{1}}^{(1) \mathrm{I}} B_{1 n_{2}}^{(1) \mathrm{I}}+C_{1 n_{1}}^{(1) \mathrm{I}} C_{1 n_{2}}^{(1) \mathrm{I}}\right] M_{k, 1,1}^{r, n_{1}, n_{2}, 1,1}
\end{aligned}
$$

from eq.(D8). The second and the third terms on the right-hand side of eq.(D12) correspond to $J\left(f_{1}^{(1)}, f_{2}^{(1)}\right)$ in the integral equation (6). Thus, it had been believed that eq. (D12) should be considered for all even $k$ because the contribution from the right-hand side of eq.(6) would not become zero even when $\Omega_{k r}^{\mathrm{H}}$ which corresponds to the first term on the right-hand side of eq.(6) is zero. 15] However, for $k=4,6$ and 8, we have confirmed that the second and the third terms on the right hand side of eq.(D12) disappear, which leads to the fact that it is not necessary to consider eq.(D12) for $k=4,6$ and 8 . Therefore, it is natural to expect that we do not need to consider eq.(D12) for even $k$ furthermore. Our contention that the second and the third terms on the right hand side of eq.(D12) will disappear for $k=2 n$ with the integer $n \geq 2$ should be demonstrated by a mathematical proof in the future. It should be mentioned that the second and the third terms on the right-hand side of eq.(D12) do not appear for odd $k$, and $\Omega_{k r}^{\mathrm{H}}$ to second order is found to be zero for odd $k$, so that we do not need to calculate $\Delta_{k r}^{\mathrm{H}}$ for odd $k$; it is not necessary to consider the case in which contribution from the right-hand side of eq.(66) becomes zero. [2]

Now, we need to consider eq.(D12) only for $k=0$ and 2. If $k=0$, eq.(D12) leads to simultaneous equations to determine the second-order coefficients $B_{0 n_{1}}^{\mathrm{II}}$ :

$$
\begin{aligned}
\Omega_{0 r}^{\mathrm{H}} & =\sum_{n_{1} \geq 2} B_{0 n_{1}}^{\mathrm{II}} M_{0,0,0}^{r, n_{1}, 0,0,0}+\sum_{n_{1}, n_{2}} B_{1 n_{1}}^{\mathrm{I}} B_{1 n_{2}}^{\mathrm{I}} M_{0,1,1}^{r, n_{1}, n_{2}, 0,0} \\
& +\sum_{n_{1}, n_{2}}\left[B_{1 n_{1}}^{(1) \mathrm{I}} B_{1 n_{2}}^{(1) \mathrm{I}}+C_{1 n_{1}}^{(1) \mathrm{I}} C_{1 n_{2}}^{(1) \mathrm{I}}\right] M_{0,1,1}^{r, n_{1}, n_{2}, 1,1}
\end{aligned}
$$

that is,

$$
\begin{array}{r}
B_{0 n_{1}}^{\mathrm{II}}=\sum_{r \geq 2}\left\{\Omega_{0 r}^{\mathrm{H}}-\sum_{n_{1}, n_{2}} B_{1 n_{1}}^{\mathrm{I}} B_{1 n_{2}}^{\mathrm{I}} M_{0,1,1}^{r, n_{1}, n_{2}, 0,0}\right. \\
\left.-\sum_{n_{1}, n_{2}}\left[B_{1 n_{1}}^{(1) \mathrm{I}} B_{1 n_{2}}^{(1) \mathrm{I}}+C_{1 n_{1}}^{(1) \mathrm{I}} C_{1 n_{2}}^{(1) \mathrm{I}}\right] M_{0,1,1}^{r, n_{1}, n_{2}, 1,1}\right\}\left(M_{0,0,0}^{r, n_{1}, 0,0,0}\right)^{-1},
\end{array}
$$

with $\Omega_{0 r}^{\mathrm{H}}$ in eqs.(D1), (D2) and (D3). We should derive the second-order coefficients $B_{0 n_{1}}^{\mathrm{II}}$ only for $n_{1} \geq 2$, because $B_{00}=1$ and $B_{01}=0$ from eq.(20). We have calculated the 
matrix $M_{0,0,0}^{r, n_{1}, 0,0,0}$ for $2 \leq r \leq 6$ and $2 \leq n_{1} \leq 6$ from eq.(D9), and we have confirmed that $M_{0,0,0}^{r, n_{1}, 0,0,0}$ vanishes for $r=0,1$ and $2 \leq n_{1} \leq 6$. We have also calculated the matrices $M_{0,1,1}^{r, n_{1}, n_{2}, 0,0}$ for $2 \leq r \leq 6,1 \leq n_{1} \leq 7$ and $1 \leq n_{2} \leq 7$ from eq.(D10), and we have confirmed $M_{0,1,1}^{r, n_{1}, n_{2}, 0,0}$ vanishes for $r=0,1,1 \leq n_{1} \leq 7$ and $1 \leq n_{2} \leq 7$. Our results for $M_{0,0,0}^{r, n_{1}, 0,0,0}$ for $2 \leq r \leq 6$ and $2 \leq n_{1} \leq 6$ and $M_{0,1,1}^{r, n_{1}, n_{2}, 0,0}$ for $2 \leq r \leq 6,1 \leq n_{1} \leq 7$ and $1 \leq n_{2} \leq 7$ are given in ref.[42]. The matrices $M_{0,1,1}^{r, n_{1}, n_{2}, 1,1}$ for $2 \leq r \leq 6,1 \leq n_{1} \leq 7$ and $1 \leq n_{2} \leq 7$, which can be calculated from eq.(D11), are also obtained from the symmetric relation $M_{0,1,1}^{r, n_{1}, n_{2}, 0,0}=M_{0,1,1}^{r, n_{1}, n_{2}, 1,1}$ arising from properties of the spherical harmonic function. Finally, we can determine the second-order coefficients $B_{0 n_{1}}^{\mathrm{II}}$ in $f_{1}$, i.e. $B_{0 r}^{\mathrm{II}}$ in eq.(14) as in eq. (37).

If $k=2$, eq.(D12) leads to simultaneous equations to determine the second-order coefficients $B_{2 n_{1}}^{\mathrm{II}}$ :

$$
\begin{aligned}
\Omega_{2 r}^{\mathrm{H}} & =\sum_{n_{1} \geq 0}\left[B_{2 n_{1}}^{\mathrm{II}} M_{2,2,0}^{r, n_{1}, 0,0,0}+B_{0 n_{1}}^{\mathrm{II}} M_{2,0,0}^{r, n_{1}, 0,0,0}\right]+\sum_{n_{1}, n_{2}} B_{1 n_{1}}^{\mathrm{I}} B_{1 n_{2}}^{\mathrm{I}} M_{2,1,1}^{r, n_{1}, n_{2}, 0,0} \\
& +\sum_{n_{1}, n_{2}}\left[B_{1 n_{1}}^{(1) \mathrm{I}} B_{1 n_{2}}^{(1) \mathrm{I}}+C_{1 n_{1}}^{(1) \mathrm{I}} C_{1 n_{2}}^{(1) \mathrm{I}}\right] M_{2,1,1}^{r, n_{1}, n_{2}, 1,1}
\end{aligned}
$$

that is,

$$
\begin{array}{r}
B_{2 n_{1}}^{\mathrm{II}}=\sum_{r \geq 0}\left\{\Omega_{2 r}^{\mathrm{H}}-\sum_{n_{1}, n_{2}} B_{1 n_{1}}^{\mathrm{I}} B_{1 n_{2}}^{\mathrm{I}} M_{2,1,1}^{r, n_{1}, n_{2}, 0,0}\right. \\
\left.-\sum_{n_{1}, n_{2}}\left[B_{1 n_{1}}^{(1) \mathrm{I}} B_{1 n_{2}}^{(1) \mathrm{I}}+C_{1 n_{1}}^{(1) \mathrm{I}} C_{1 n_{2}}^{(1) \mathrm{I}}\right] M_{2,1,1}^{r, n_{1}, n_{2}, 1,1}\right\}\left(M_{2,2,0}^{r, n_{1}, 0,0,0}\right)^{-1},
\end{array}
$$

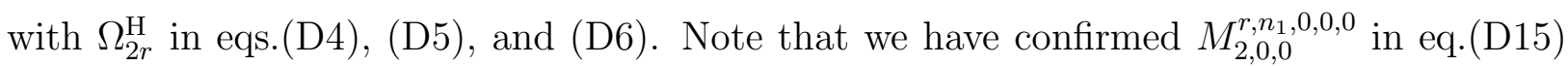
becomes zero, which had been also confirmed by Burnett 15]. We should derive the secondorder coefficients $B_{2 n_{1}}^{\mathrm{II}}$ for $n_{1} \geq 0$. We have calculated the matrix $M_{2,2,0}^{r, n_{1}, 0,0,0}$ for $0 \leq r \leq 6$ and $0 \leq n_{1} \leq 6$ from eq.(D9), and also the matrices $M_{2,1,1}^{r, n_{1}, n_{2}, 0,0}$ for $0 \leq r \leq 6,1 \leq n_{1} \leq 7$ and $1 \leq n_{2} \leq 7$ from eq.(D10). Our results are given in ref. 42]. The matrices $M_{2,1,1}^{r, n_{1}, n_{2}, 1,1}$ for $0 \leq r \leq 6,1 \leq n_{1} \leq 7$ and $1 \leq n_{2} \leq 7$, which can be calculated from eq.(D11), are also obtained from the symmetric relation $M_{2,1,1}^{r, n_{1}, n_{2}, 0,0}=-2 M_{2,1,1}^{r, n_{1}, n_{2}, 1,1}$ arising from properties of the spherical harmonic function. 15] The second-order coefficients $B_{k_{1} n_{1}}^{\mathrm{II}}$ in $f_{1}$, i.e. $B_{k r}^{\mathrm{II}}$ in eq.(16) can be written in the final form shown in eq.(38). 


\section{APPENDIX E: TEST OF THE NONEQUILIBRIUM STEADY-STATE THER-} MODYNAMICS

We examine SST from the microscopic viewpoint, that is, by applying the velocity distribution function for the steady-state Boltzmann equation for both hard-core molecules and Maxwell molecules to the cell on the right-hand side in a simple nonequilibrium steady-state system illustrated in Fig 4. We also use the velocity distribution function for the steady-state BGK equation to second order in the test of SST. This simple nonequilibrium steady-state system is inspired by SST suggested by Sasa and Tasaki[46]. They predicted the following results. When the system shown in Fig 4 is in a steady-state, the osmosis, defined as the difference between the pressure $P_{x x}$ of the cell in the nonequilibrium steady state and the value $P_{0}$ of the cell at equilibrium, namely

$$
\Delta P \equiv P_{x x}-P_{0}
$$

always becomes positive. Additionally, there is a relation

$$
\frac{n(0)}{n_{0}}=\left(\frac{\partial P_{x x}}{\partial P_{0}}\right)_{T_{0}, J_{x}}
$$

connecting $n(0), P_{x x}, n_{0}$ and $P_{0}$, where $n(0)$ is the density of the cell in the nonequilibrium steady state around the hole and $n_{0}$ is that of the cell at equilibrium.

We consider the nonequilibrium steady-state where the mean mass flux at the hole is zero:

$$
\int_{0}^{\infty} \mathrm{d} c_{x} \int_{-\infty}^{\infty} \mathrm{d}^{2} c_{\perp} m c_{x} f_{0}+\left.\int_{-\infty}^{0} \mathrm{~d} c_{x} \int_{-\infty}^{\infty} \mathrm{d}^{2} c_{\perp} m c_{x} f\right|_{x=0}=0
$$

where $c_{\perp}$ represents the components of the velocity which are orthogonal to $c_{x}$, i.e. $c_{y}$ and $c_{z}$. Note that we consider the mean mass flux at the hole, so that we put $x=0$ in f. $f_{0}=n_{0}\left(m / 2 \pi \kappa T_{0}\right)^{3 / 2} e^{-m \mathbf{v}^{2} / 2 \kappa T_{0}}$ is the velocity distribution function of the cell on the left-hand side at equilibrium at temperature $T_{0}$ and density $n_{0}$. From eq.(E3), the relation between $n(0)$ and $n_{0}$ is obtained as

$$
n(0)=n_{0}\left[1+\lambda_{n} \frac{m J_{x}^{2}}{n_{0}^{2} \kappa^{3} T_{0}^{3}}\right]
$$

to second order. The value for the constant $\lambda_{n}$ is given in Table $\nabla$. The density of the cell in the nonequilibrium steady state around the hole $n(0)$ is greater than that of the cell at 
equilibrium $n_{0}$ regardless of the sign of $J_{x}$. We emphasize that $\lambda_{n}$ could not be calculated if we did not derive the explicit form of the velocity distribution function $f$ to second order. We have adopted the boundary condition around the hole, $T(0)=T_{0}$, in order to examine SST. Although it is believed that the Knudsen layer effect, i.e. the slip effect is dominant around the 'wall' [5, 16, 21], for reasons of simplification, we do not consider the slip effect around the 'hole' in this paper. If the slip effect is not dominant, the density of the cell in the nonequilibrium steady state will always be larger than that of the cell at equilibrium, regardless of the sign of $J_{x}$. This can be tested by experiments on the nonequilibrium steady-state system shown in Fig, 4

We also calculate the osmosis $\Delta P$ in eq.(E1) as

$$
\Delta P=\lambda_{\Delta P} \frac{m J_{x}^{2}}{n_{0} \kappa^{2} T_{0}^{2}},
$$

to second order, using eqs.(43), (E4) and $P_{0}=n_{0} \kappa T_{0}$. Values for $\lambda_{\Delta P}$ are given in Table $\mathrm{V}$, We have found that $\lambda_{\Delta P}$ is always positive, which agrees with the prediction by SST [46]. Furthermore, we are able to test the relation (E2). Though substitution of eqs.(43) and (E4) into eq.(E2) leads to the relation $\lambda_{P}^{x x} / \lambda_{n}=-2$, our numerical results conflict with this relation: our results give $\lambda_{P}^{x x} / \lambda_{n}=-0.5604$ for 7 th approximation $b_{0 r}$ and $b_{2 r}$ for hard-core molecules and $\lambda_{P}^{x x} / \lambda_{n}=0$ for both Maxwell molecules and the steady-state BGK equation. We have also confirmed that the relation $\lambda_{P}^{x x} / \lambda_{n}=-2$ predicted in SST [46] is not modified if the boundary condition around the hole can be written in the form:

$$
T(0)=T_{0}\left[1+\lambda_{T} \frac{m J_{x}^{2}}{n_{0}^{2} \kappa^{3} T_{0}^{3}}\right],
$$

where the constant $\lambda_{T}$ represents the difference between the temperature of molecules around the hole and that of the mid-wall. Our boundary condition $T(0)=T_{0}$ corresponds to putting $\lambda_{T}=0$ for any kinetic models. It can be concluded that, although we regard a state in which the mean mass flux at the hole is zero to be a nonequilibrium steady state, as in eq. (E3), this state has yet to be interpreted phenomenologically.

On the other hand, by virtue of the derivation of the explicit form of the velocity distribution function $f$ to second order, we can also calculate the $x$ component of the heat flux 
at the hole as

$$
\begin{aligned}
J^{*} & \equiv\left(\frac{2 \kappa T}{m}\right)^{3} \int_{0}^{\infty} \mathrm{d} c_{x} \int_{-\infty}^{\infty} \mathrm{d}^{2} c_{\perp} \frac{m \mathbf{c}^{2}}{2} c_{x} f_{0}+\left.\left(\frac{2 \kappa T}{m}\right)^{3} \int_{-\infty}^{0} \mathrm{~d} c_{x} \int_{-\infty}^{\infty} \mathrm{d}^{2} c_{\perp} \frac{m \mathbf{c}^{2}}{2} c_{x} f\right|_{x=0} \\
& =\frac{J_{x}}{2}+\lambda_{J^{*}} \frac{m J_{x}^{2}}{n_{0} \kappa^{2} T_{0}^{2}}\left(\frac{2 \kappa T_{0}}{\pi m}\right)^{\frac{1}{2}},
\end{aligned}
$$

to second order using eq.(E4). Numerical values for the constant $\lambda_{J^{*}}$ are given in Table V] The first-order term on the right-hand side of eq.(E7) is from Fourier's law, while the second-order term also appears on the right-hand side of eq. (E7), though the second-order heat flux $\mathbf{J}^{(2)}$ does not exist in the cell in the nonequilibrium steady state. This fact indicates that $T(0)$ is not appropriate for the nonequilibrium temperature, if $J^{*}$ suggests the existence of the nonequilibrium temperature. 
[1] S. Chapman and T. G. Cowling: The Mathematical Theory of Non-uniform Gases(Cambridge University Press, London, 1970) 3rd ed.

[2] P. Résibois and M. De Leener: Classical Kinetic Theory of Fluids(A Wiley-Interscience Publication, New York, 1977).

[3] M. N. Kogan: Rarefied Gas Dynamics(Plenum Press, New York, 1969).

[4] J. A. Mclennan: Introduction to Nonequilibrium Statistical Mechanics(Prentice Hall, Englewood Cliffs, New Jersey, 1990). This book can be obtained from http://www.lehigh.edu/ ljm3/ljm3.html

[5] C. Cercignani: Mathematical Methods in Kinetic Theory(Plenum Press, New York, 1990).

[6] S. Flügge: Thermodynamics of Gases(Springer-Verlag, Berlin, 1958).

[7] J. L. Lebowitz, E. W. Montroll: The Boltzmann equation(Elsevier Science, New York, 1983).

[8] E. G. D. Cohen and W. Thirring: The Boltzmann equation : theory and applications(SpringerVerlag, Berlin, 1973).

[9] C. Cercignani: The Boltzmann equation and its applications(Springer-Verlag, Berlin, 1988).

[10] C. Cercignani: Theory and application of the Boltzmann equation(Chatto and Windus, London, 1975).

[11] V. V. Aristov: Direct Method for Solving the Boltzmann equation and Study of Nonequilibrium Flows(Kluwer Academic Publishers, Dordrecht, 2001).

[12] J. C. Maxwell: Collected papers 2 (1890) 42.

[13] J. C. Maxwell: Phil. Trans. R. Soc. 157 (1867) 49.

[14] E. Ikenberry and C. Truesdell: J. Rational Mech. Anal. 5 (1956) 1.

[15] D. Burnett: Proc. Lond. Math. Soc. 40 (1935) 382.

[16] R. Schamberg: Ph. D thesis, California Institute of Technology (1947).

[17] M. Sh. Shavaliev: J. Appl. Math. Mech. 42 (1978) 698.

[18] S. Reinecke and G. M. Kremer: Continuum Mech. Thermodyn. 3 (1991) 155.

[19] S. Reinecke and G. M. Kremer: Continuum Mech. Thermodyn. 8 (1996) 121.

[20] I. V. Karlin and A. N. Gorban: Annalen der Physik 11 (2002) 783.

[21] C. Cercignani: University of California Report No. AS-64-19, (1964).

[22] P. L. Bhatnagar, E. P. Gross and M. Krook: Phys. Rev. 94 (1954) 511. 
[23] J. L. Lebowitz: Phys. Rev. 114 (1959) 1192.

[24] Y. J. Park and C. S. Kim: J. Korean Phys. Soc. 29 (1996) 466.

[25] F. Bouchut and B. Perthame: J. Stat. Phys.71 (1993) 191.

[26] A. Santos, J. J. Brey, C. S. Kim and J. W. Dufty: Phys. Rev. A 39 (1989) 320.

[27] C. S. Kim, J. W. Dufty, A. Santos and J. J. Brey: Phys. Rev. A 39 (1989) 328.

[28] C. S. Kim, J. W. Dufty, A. Santos and J. J. Brey: Phys. Rev. A 40 (1989) 7165.

[29] A. Santos and J. J. Brey: Physica A, 174 (1991) 355.

[30] J. J. Brey, A. Santos and J. W. Dufty: Phys. Rev. A 36 (1987) 2842.

[31] M. Tij and A. Santos: Phys. Fluids 7 (1995) 2858.

[32] J. M. Montanero and A. Santos: Physica A 225 (1996) 7.

[33] A. Santos: Phys. Rev. E 62 (2000) 6597.

[34] J. D. Foch Jr.: Acta Phys. Aust. Suppl. X (1973) 123.

[35] K. A. Fiscko and D. R. Chapman: Rarefied Gas Dynamics Mechanics of Nonequilibrium Processes, ed. E. P. Muntz, D. P. Weaver and D.H. Campbell(American Institute of Aeronautics and Astronautics, Washington, D.C., 1989), p.374.

[36] F. E. Lumpkin III and D. R. Chapman: J. Thermophys. Heat Trans. 6 (1992) 419.

[37] V. S. Galkin and V. I. Nosik: Izv. Ross. Akad. Nauk. MZhG 3 (1999) 126.

[38] J. Popielawski: J. Chem. Phys. 83 (1985) 790.

[39] C. S. Wang-Chang and G. E. Uhlenbeck: Studies in Statistical Mechanics, ed. J. de Boer and G. E. Uhlenbeck (North-Holland, Amsterdam, 1970) Vol. V, p.1.

[40] J. Fort and A. S. Cukrowski: Chem. Phys. 222 (1997) 59.

[41] Kim H.-D. and H. Hayakawa: Chem. Phys. Lett. 372 (2003) 314.

[42] http://ace.phys.h.kyoto-u.ac.jp/ ${ }^{\sim}$ kim/supplement.html

[43] S. Chapman: Mem. and Proc. Manchester Lit. and Phil. Soc. 66 (1922).

[44] D. Alonso, R. Artuso, G. Casati and I. Guarneri: Phys. Rev. Lett. 82 (1999) 1859.

[45] Y. Oono and M. Paniconi: Prog. Theor. Phys. Suppl. 130 (1998) 29.

[46] S. Sasa and H. Tasaki: cond-mat/0108365.

[47] R. D. Present: Kinetic Theory of Gases(Mcgraw-Hill, New York, 1958).

[48] Kim H.-D. and H. Hayakawa: cond-mat/0203036, submitted to J. Phys. Soc. Jpn.

[49] Please contact Kim H.-D. by e-mail. We have provided a technical note about our calculation. See also ref.[42]. 
TABLE I: Numerical constants $b_{1 r}$ in eq.(33)

\begin{tabular}{cccccc}
\hline$r$ & $r \leq 4$ & $r \leq 5$ & $r \leq 6$ & $r \leq 7$ & Maxwell's $b_{1 r}$ \\
\hline 1 & 1.025 & 1.025 & 1.025 & 1.025 & 1 \\
\hline $24.881 \times 10^{-2}$ & $4.889 \times 10^{-2}$ & $4.891 \times 10^{-2}$ & $4.892 \times 10^{-2}$ & 0 \\
\hline $33.639 \times 10^{-3}$ & $3.698 \times 10^{-3}$ & $3.711 \times 10^{-3}$ & $3.715 \times 10^{-3}$ & 0 \\
\hline $42.526 \times 10^{-4}$ & $2.838 \times 10^{-4}$ & $2.905 \times 10^{-4}$ & $2.922 \times 10^{-4}$ & 0 \\
\hline 5 & - & $1.855 \times 10^{-5}$ & $2.123 \times 10^{-5}$ & $2.187 \times 10^{-5}$ & 0 \\
\hline 6 & - & - & $1.284 \times 10^{-6}$ & $1.492 \times 10^{-6}$ & 0 \\
\hline 7 & - & - & - & $8.322 \times 10^{-8}$ & 0 \\
\hline
\end{tabular}

TABLE II: Numerical constants $b_{0 r}$ in eq.(37)

\begin{tabular}{cccccc}
\hline$r$ & $r \leq 4$ & $r \leq 5$ & $r \leq 6$ & $r \leq 7$ & Maxwell's $b_{0 r}$ \\
\hline 2 & $4.434 \times 10^{-1}$ & $4.390 \times 10^{-1}$ & $4.381 \times 10^{-1}$ & $4.380 \times 10^{-1}$ & $\frac{825}{1024}$ \\
\hline 3 & $-4.935 \times 10^{-2}$ & $-5.342 \times 10^{-2}$ & $-5.413 \times 10^{-2}$ & $-5.429 \times 10^{-2}$ & $-\frac{25}{256}$ \\
\hline 4 & - & $-3.581 \times 10^{-3}$ & $-4.007 \times 10^{-3}$ & $-4.098 \times 10^{-3}$ & 0 \\
\hline 5 & - & - & $-2.779 \times 10^{-4}$ & $-3.184 \times 10^{-4}$ & 0 \\
\hline 6 & - & - & - & $-2.087 \times 10^{-5}$ & 0 \\
\hline
\end{tabular}


TABLE III: Numerical constants $b_{2 r}$ in eq. (38)

\begin{tabular}{|c|c|c|c|c|c|}
\hline$r$ & $r \leq 4$ & $r \leq 5$ & $r \leq 6$ & $r \leq 7$ & Maxwell's $b_{2 r}$ \\
\hline 0 & $-3.353 \times 10^{-2}$ & $-3.327 \times 10^{-2}$ & $-3.322 \times 10^{-2}$ & $-3.320 \times 10^{-2}$ & 0 \\
\hline 1 & $-1.285 \times 10^{-1}$ & $-1.278 \times 10^{-1}$ & $-1.277 \times 10^{-1}$ & $-1.276 \times 10^{-1}$ & $\frac{75}{896}$ \\
\hline 2 & $6.320 \times 10^{-1}$ & $6.394 \times 10^{-2}$ & $6.410 \times 10^{-2}$ & $6.414 \times 10^{-2}$ & $\frac{125}{1536}$ \\
\hline 3 & $4.884 \times 10^{-3}$ & $5.395 \times 10^{-3}$ & $5.496 \times 10^{-3}$ & $5.521 \times 10^{-3}$ & 0 \\
\hline 4 & - & $3.609 \times 10^{-4}$ & $4.101 \times 10^{-4}$ & $4.214 \times 10^{-4}$ & 0 \\
\hline 5 & - & - & $2.685 \times 10^{-5}$ & $3.106 \times 10^{-5}$ & 0 \\
\hline 6 & - & - & - & $1.861 \times 10^{-6}$ & 0 \\
\hline
\end{tabular}

TABLE IV: Numerical constants for the macroscopic quantities I: the $i$ th approximation quantities for hard-core molecules and the exact values for Maxwell molecules and the steady-state BGK equation.

\begin{tabular}{|c|c|c|c|c|c|}
\hline$i$ th & $\lambda_{P}^{x x}$ & $\lambda_{P}^{y y}$ & $\lambda_{T_{x}}$ & $\lambda_{T_{y}}$ & $\lambda_{S}$ \\
\hline 4 th & $-4.647 \times 10^{-2}$ & $2.324 \times 10^{-2}$ & $-2.324 \times 10^{-2}$ & $1.162 \times 10^{-2}$ & $-2.034 \times 10^{-1}$ \\
\hline 5 th & $-4.610 \times 10^{-2}$ & $2.305 \times 10^{-2}$ & $-2.305 \times 10^{-2}$ & $1.153 \times 10^{-2}$ & $-2.035 \times 10^{-1}$ \\
\hline 6 th & $-4.602 \times 10^{-2}$ & $2.301 \times 10^{-2}$ & $-2.301 \times 10^{-2}$ & $1.151 \times 10^{-2}$ & $-2.035 \times 10^{-1}$ \\
\hline 7th & $-4.600 \times 10^{-2}$ & $2.300 \times 10^{-2}$ & $-2.300 \times 10^{-2}$ & $1.150 \times 10^{-2}$ & $-2.035 \times 10^{-1}$ \\
\hline Maxwell & 0 & 0 & 0 & 0 & $-\frac{1}{5}$ \\
\hline BGK equation & 0 & 0 & 0 & 0 & $-\frac{1}{5}$ \\
\hline
\end{tabular}


TABLE V: Numerical constants for the macroscopic quantities II: the $i$ th approximation quantities for hard-core molecules and the exact values for Maxwell molecules and the steady-state BGK equation. The values of $\lambda_{n}, \lambda_{\Delta P}$ and $\lambda_{J^{*}}$ for hard-core molecules has not yet converged to 4 th approximation $b_{0 r}$ and $b_{2 r}$ values. The ratios of the 7 th to the 6 th approximation $\lambda_{n}, \lambda_{\Delta P}$ and $\lambda_{J^{*}}$ are 1.011, 1.023 and 1.004, respectively, so that the errors included in the 7 th approximation $\lambda_{n}$, $\lambda_{\Delta P}$ and $\lambda_{J^{*}}$ appear to be less than about one or two percent. Note that $n(0)=n_{0}$ to first order, and that osmosis does not appear to first order.

\begin{tabular}{cccc}
\hline$i$ th & $\lambda_{n}$ & $\lambda_{\Delta P}$ & $\lambda_{J^{*}}$ \\
\hline 4 th & $9.255 \times 10^{-2}$ & $4.608 \times 10^{-2}$ & $-3.237 \times 10^{-1}$ \\
\hline 5 th & $8.528 \times 10^{-2}$ & $3.917 \times 10^{-2}$ & $-3.109 \times 10^{-1}$ \\
\hline 6 th & $8.296 \times 10^{-2}$ & $3.694 \times 10^{-2}$ & $-3.073 \times 10^{-1}$ \\
\hline 7 th & $8.210 \times 10^{-2}$ & $3.609 \times 10^{-2}$ & $-3.060 \times 10^{-1}$ \\
\hline Maxwell & $\frac{71}{1575}$ & $\frac{71}{1575}$ & $-\frac{41}{105}$ \\
\hline BGK equation & $\frac{2}{25}$ & $\frac{2}{25}$ & $-\frac{11}{25}$ \\
\hline
\end{tabular}

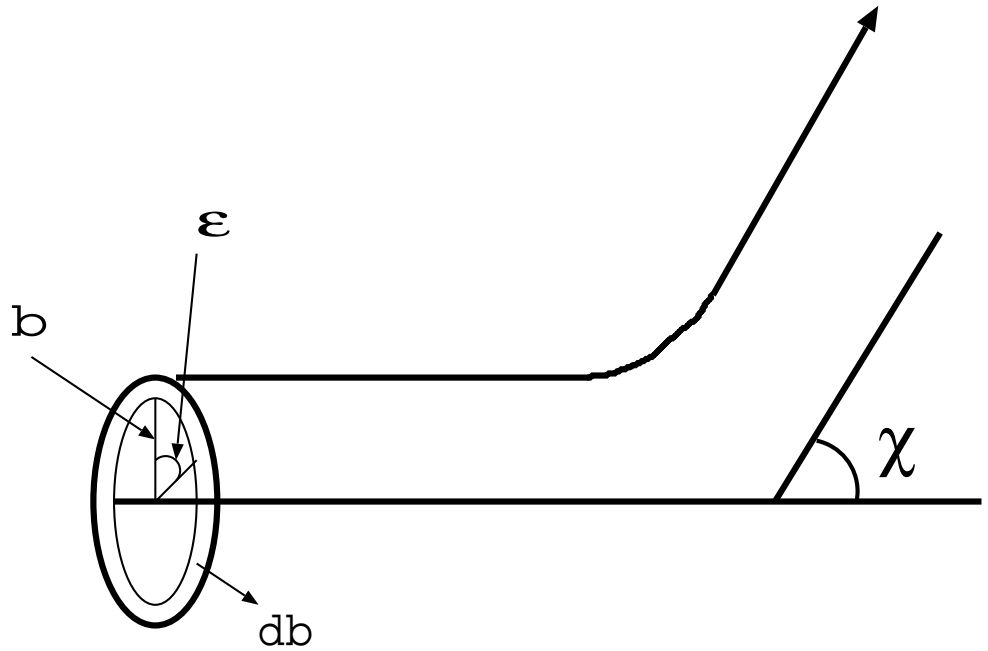

FIG. 1: Schematic description of an interaction. 


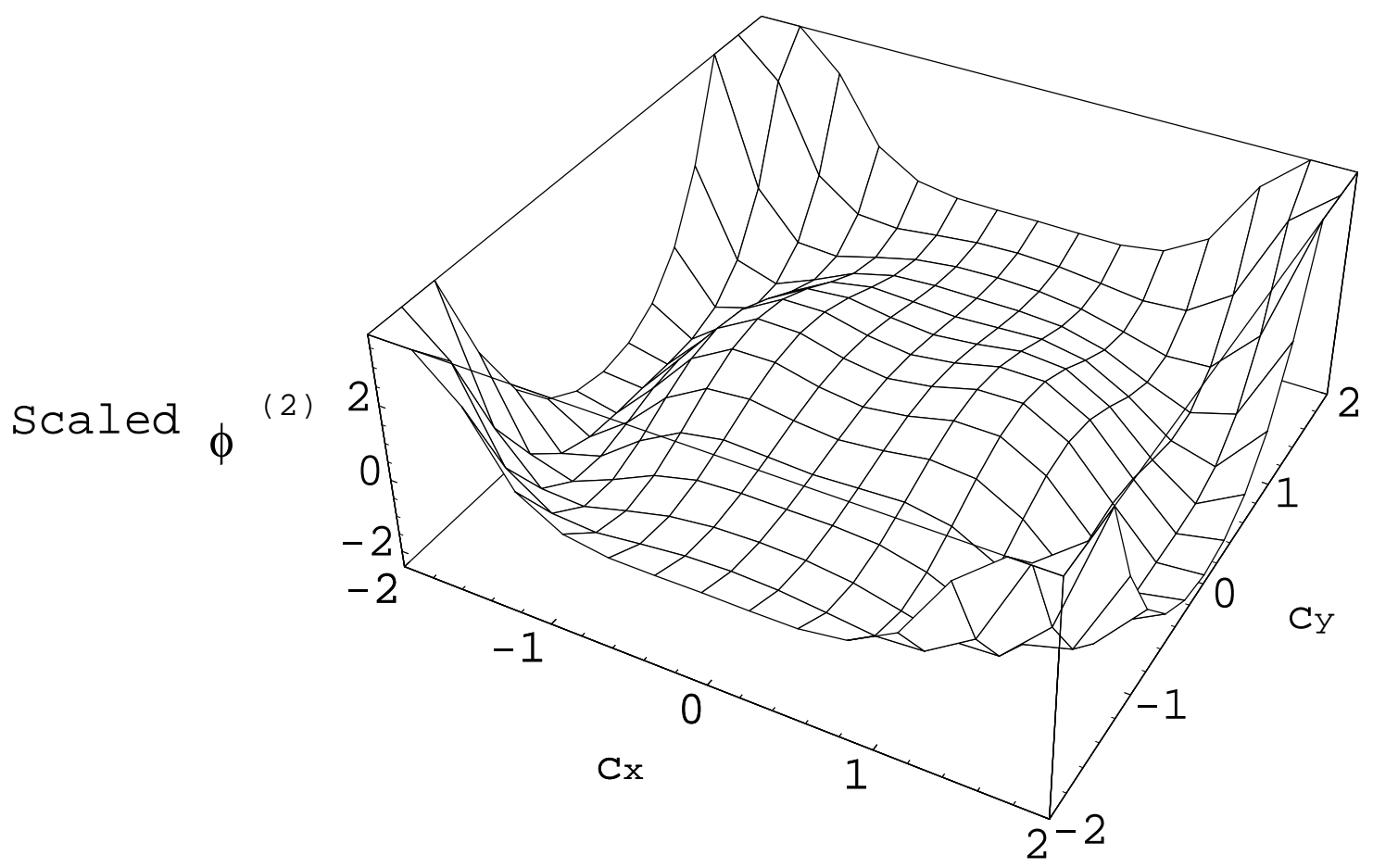

FIG. 2: The scaled $\phi^{(2)}$ for hard-core molecules with 7th approximation $b_{0 r}$ and $b_{2 r}$. Note that we put $c_{z}=0$. 


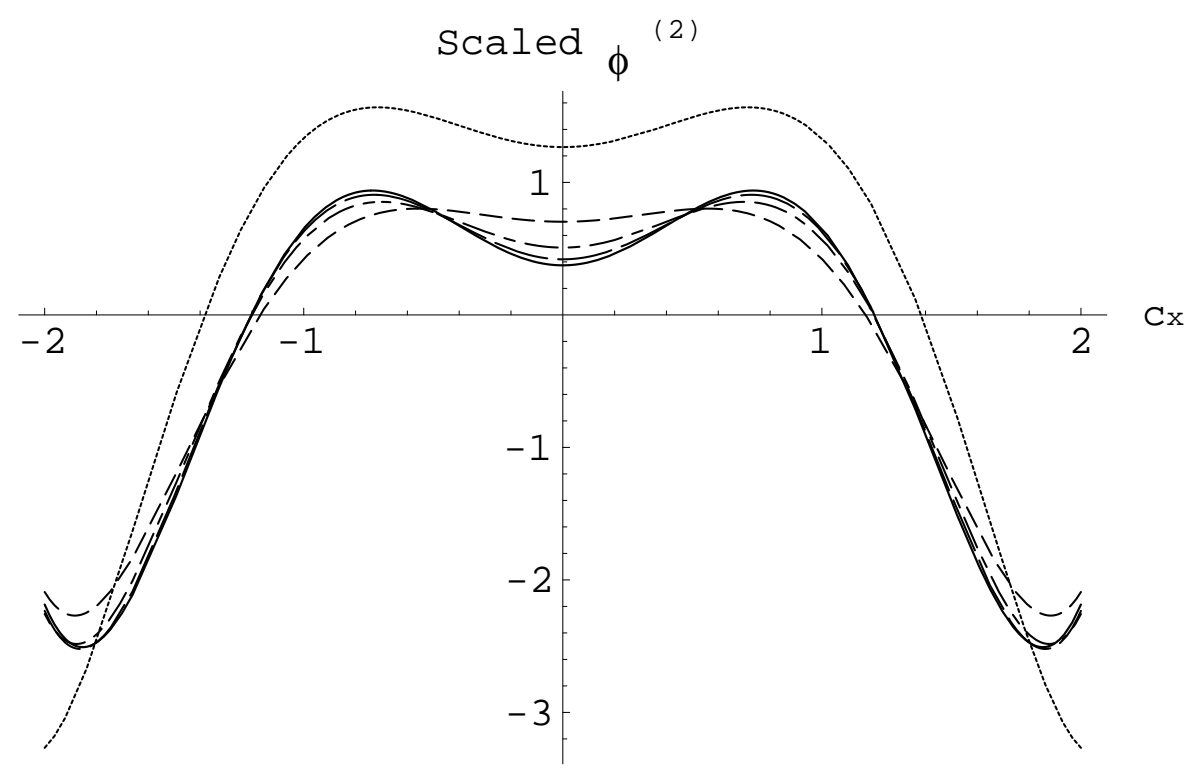

FIG. 3: Comparison of the scaled $\phi^{(2)}$ s for hard-core molecules with the scaled $\phi^{(2)}$ for Maxwell molecules. The dashed line, the dash-dotted line, the long-dashed line and the solid line correspond to the scaled $\phi^{(2)} \mathrm{s}$ for hard-core molecules with $4 \mathrm{th}, 5 \mathrm{th}, 6$ th and 7 th approximation $b_{0 r} \mathrm{~s}$ and $b_{2 r} \mathrm{~s}$, respectively. The dotted line is the scaled $\phi^{(2)}$ for Maxwell molecules. Note that we put $c_{y}=c_{z}=0$. 


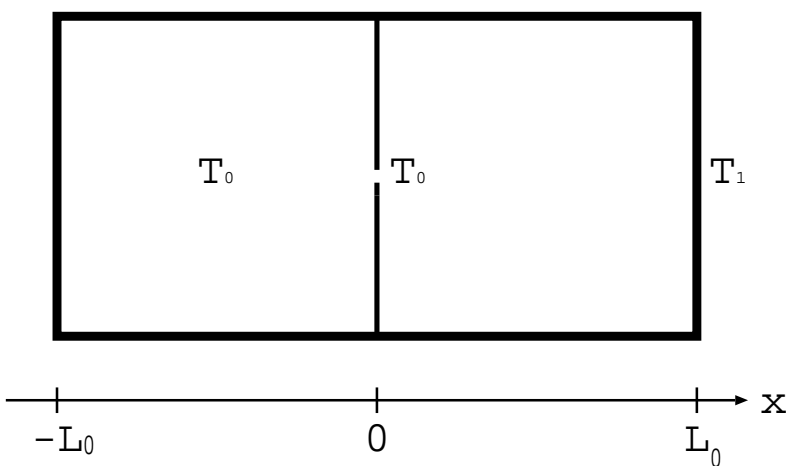

FIG. 4: The cell on the left-hand side is at equilibrium at temperature $T_{0}$. The cell on the righthand side is in a nonequilibrium state under a temperature gradient along the $x$-axis caused by the right wall (at $x=L_{0}$ ) at temperature $T_{1}$ and the thin mid-wall (at $x=0$ ) at temperature $T_{0}$, of thickness less than or equal to the mean free path $l$ of the dilute gases. Both cells are filled with dilute gases and connected by a small hole of diameter $d$ on the thin mid-wall. The diameter of the small hole $d$ is much smaller than $l$, i.e. $d \ll l$. Molecules which have passed through the small hole are relaxed into the state of the cell they go into after a few interactions, so that they do not affect the macroscopic state of that cell. The mean mass flux at the small hole is zero in the nonequilibrium steady state. 